\title{
The Role of Neuroglial Crosstalk and Synaptic Plasticity-Mediated Central Sensitization in Acupuncture Analgesia
}

\author{
Zhongxi Lyu, ${ }^{1,2,3}$ Yongming Guo $\mathbb{D},{ }^{1,2,3}$ Yinan Gong $\mathbb{D}^{1}$, Wen Fan $\mathbb{D},{ }^{1,4}$ Baomin Dou, ${ }^{1}$ \\ Ningcen Li, ${ }^{1}$ Shenjun Wang, ${ }^{1,2,3}$ Yuan $\mathrm{Xu},{ }^{1,2,3}$ Yangyang Liu $\mathbb{D}^{1},{ }^{1,2,3}$ Bo Chen $\mathbb{D}^{1,2,3}$ \\ Yi Guo $\mathbb{D}^{1,3,5}$ Zhifang $X u \mathbb{D}^{1,2,3}$ and Xiaowei Lin $\mathbb{D}^{1,3,5}$ \\ ${ }^{1}$ Research Center of Experimental Acupuncture Science, Tianjin University of Traditional Chinese Medicine, Tianjin 301617, China \\ ${ }^{2}$ School of Acupuncture \& Moxibustion and Tuina, Tianjin University of Traditional Chinese Medicine, Tianjin 301617, China \\ ${ }^{3}$ National Clinical Research Center for Chinese Medicine Acupuncture and Moxibustion, Tianjin 300381, China \\ ${ }^{4}$ Suzuka University of Medical Science, Suzuka 5100293, Japan \\ ${ }^{5}$ School of Traditional Chinese Medicine, Tianjin University of Traditional Chinese Medicine, Tianjin 301617, China
}

Correspondence should be addressed to Zhifang Xu; xuzhifangmsn@hotmail.com and Xiaowei Lin; linxiaoweiwqhz@163.com

Zhongxi Lyu and Yongming Guo contributed equally to this work.

Received 23 June 2020; Revised 30 December 2020; Accepted 7 January 2021; Published 18 January 2021

Academic Editor: Zhen Zheng

Copyright (C) 2021 Zhongxi Lyu et al. This is an open access article distributed under the Creative Commons Attribution License, which permits unrestricted use, distribution, and reproduction in any medium, provided the original work is properly cited.

\begin{abstract}
Although pain is regarded as a global public health priority, analgesic therapy remains a significant challenge. Pain is a hypersensitivity state caused by peripheral and central sensitization, with the latter considered the culprit for chronic pain. This study summarizes the pathogenesis of central sensitization from the perspective of neuroglial crosstalk and synaptic plasticity and underlines the related analgesic mechanisms of acupuncture. Central sensitization is modulated by the neurotransmitters and neuropeptides involved in the ascending excitatory pathway and the descending pain modulatory system. Acupuncture analgesia is associated with downregulating glutamate in the ascending excitatory pathway and upregulating opioids, ?-aminobutyric acid, norepinephrine, and 5-hydroxytryptamine in the descending pain modulatory system. Furthermore, it is increasingly appreciated that neurotransmitters, cytokines, and chemokines are implicated in neuroglial crosstalk and associated plasticity, thus contributing to central sensitization. Acupuncture produces its analgesic action by inhibiting cytokines, such as interleukin- $\beta$, interleukin-6, and tumor necrosis factor- $\alpha$, and upregulating interleukin-10, as well as modulating chemokines and their receptors such as CX3CL1/CX3CR1, CXCL12/CXCR4, CCL2/CCR2, and CXCL1/CXCR2. These factors are regulated by acupuncture through the activation of multiple signaling pathways, including mitogen-activated protein kinase signaling (e.g., the p38, extracellular signal-regulated kinases, and c-Jun-N-terminal kinase pathways), which contribute to the activation of nociceptive neurons. However, the responses of chemokines to acupuncture vary among the types of pain models, acupuncture methods, and stimulation parameters. Thus, the exact mechanisms require future clarification. Taken together, inhibition of central sensitization modulated by neuroglial plasticity is central in acupuncture analgesia, providing a novel insight for the clinical application of acupuncture analgesia.
\end{abstract}

\section{Introduction}

Pain is mediated by nociceptive nerve fibers and is a physiological alarm response to protect the body by reducing tissue damage from injury [1]. Thirty to fifty percent of patients with pathological pain suffer from anxiety and depression long after wound healing. Both the pain and unpleasant emotions affect their quality of life and cause serious social and economic consequences $[2,3]$. However, the solution for chronic pain remains a major challenge throughout the world. Oral drugs are often the first choice, and their usage has expanded exponentially in recent years [4]. Unfortunately, the extensive application of analgesic drugs may result in organ damage and abuse, together with causing serious social problems [5]. 
For example, long-term overuse of opioids, the most common class of prescribed painkillers, can lead to side effects such as addiction, tolerance, and drowsiness, as well as impaired memory, attention, and judgment. Opioid abuse can also potentially cause respiratory depression $[6,7]$. Therefore, a natural analgesic that can also regulate pain-related moods and cognitive disorders is necessary.

Acupuncture therapy is a well-known treatment that originated in China and has been applied in 183 countries and regions all over the world $[8,9]$. Long-term clinical practice has proved that acupuncture is an effective treatment to relieve pain. The World Health Organization has recommended acupuncture for more than 30 types of pain conditions, including lumbago, headache, sciatica, and postoperative pain [10]. Referring to the National Guideline Clearinghouse (http://www.guidelines.gov/), there are 49 specific medical recommendations for acupuncture, of which 37 (75.51\%) are pain-related diseases [11]. At present, many clinical randomized controlled trials (RCTs) have demonstrated the analgesic effect of acupuncture [12]. As shown in a 16-week RCT, compared with sham acupuncture treatment and awaiting-treatment groups, acupuncture treatment could significantly reduce the incidence of migraine without premonitory migraine, an effect that lasted at least 24 weeks [13]. A meta-analysis by Vickers et al. analyzed the individual data of patients in RCTs with nonspecific musculoskeletal pain, osteoarthritis, shoulder pain, and chronic headache, concluding that acupuncture is effective for the treatment of chronic pain and has long-lasting therapeutic effects [12]. These studies have indicated that acupuncture analgesia is effective and safe and can improve the quality of life of patients suffering from pain.

Pain is caused by tissue damage or similar pathophysiological causes. Pain sensitization plays a key role in pain occurrence and maintenance. Pain sensitization starts from the sensitization of peripheral nerves and involves a series of neuroplastic changes in the spinal cord and brain $[14,15]$, namely, central sensitization. Neurotransmitters, neurotrophic factors, lipids, and cytokines/chemokines play important roles in the communication between neurons and glial cells in both peripheral and central sensitization [16, 17]. Interestingly, increasing evidence suggests that acupuncture can alleviate central sensitization induced by glial cell-mediated inflammation to achieve analgesia $[18,19]$. Therefore, we have summarized the analgesic mechanisms of acupuncture in terms of neuroglial crosstalk and neuroplasticity-mediated central sensitization, highlighting the central neuroimmunological regulating mechanisms and providing novel evidence and insights for the clinical application of acupuncture.

\section{Methods}

2.1. Search Strategy. We searched the PubMed database for published studies, from January 2010 to April 2020. The keywords included ["acupuncture" or "electroacupuncture" or "manual acupuncture"] and ["pain" or "analgesia" or "analgesic"]. The language was limited to English. The filter process was done firstly by the website's search engine which initially identified 2888 articles.

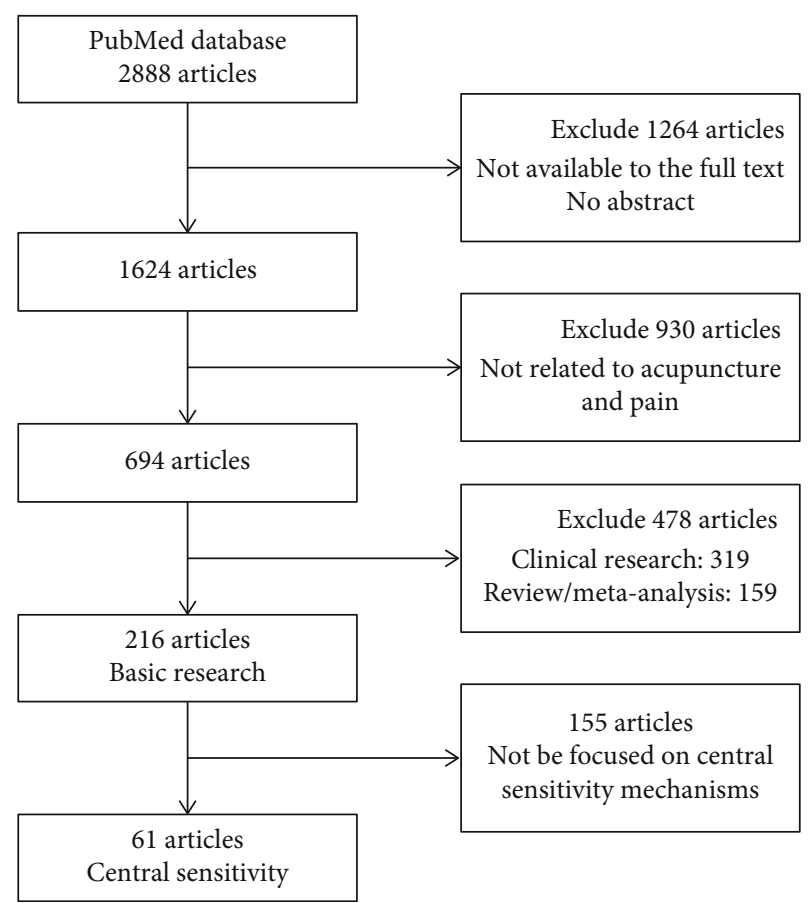

FIGURE 1: Flow chart of the search processes.

2.2. Study Selection. Of these articles, we excluded 1264 articles due to the absence of an abstract or unavailability of the full text, leaving 1624 articles. Hand searching was performed by screening the reference lists of articles that met our inclusion criteria based on the titles and abstracts. Of these, we excluded 930 articles as not related to acupuncture and pain in the titles and abstracts before full-text assessments resulting in 694 articles. These included 216 basic research articles, 319 clinical research articles, and 159 review articles or meta-analyses. The full texts of 216 basic research articles meeting the inclusion criteria were obtained and read carefully. Of these, 155 articles were excluded from 216 basic researches due to not be focused on central sensitivity mechanisms, resulting in 61 articles. A flowchart of the search process is shown in Figure 1.

2.3. Data Extraction. Of 61 basic researches on the central mechanism, the information from 35 typical and recently published studies are listed in Table 1 due to similarities in some of the studies, to analyze the central sensitizationrelated mechanisms of acupuncture analgesia. The study design data were extracted and classified using a predefined data extraction form that designated the pain model type, the intervention (methods, acupoints, acupuncture parameters), and the outcome measures (pain-related behavior, mechanism indexes). The data were extracted mainly by one author and were checked by the other authors.

\section{Role of Neural-Immune Crosstalk in Peripheral Sensitization}

The basic process of pain generation occurs when nociceptors located in the periphery of peptidergic and nonpeptidergic primary nociceptive neurons selectively respond to thermal, mechanical nociception, and irritating chemicals. 


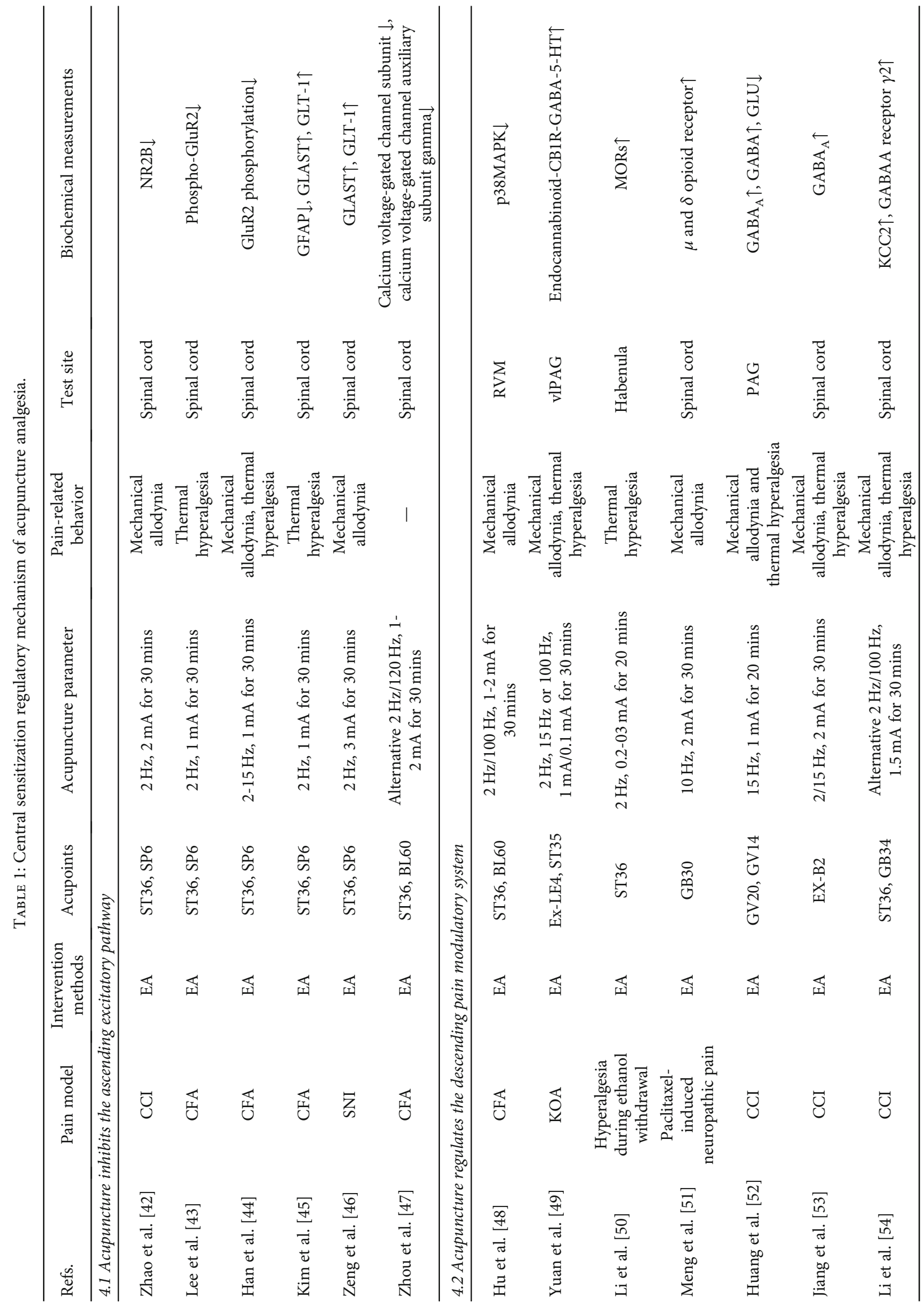




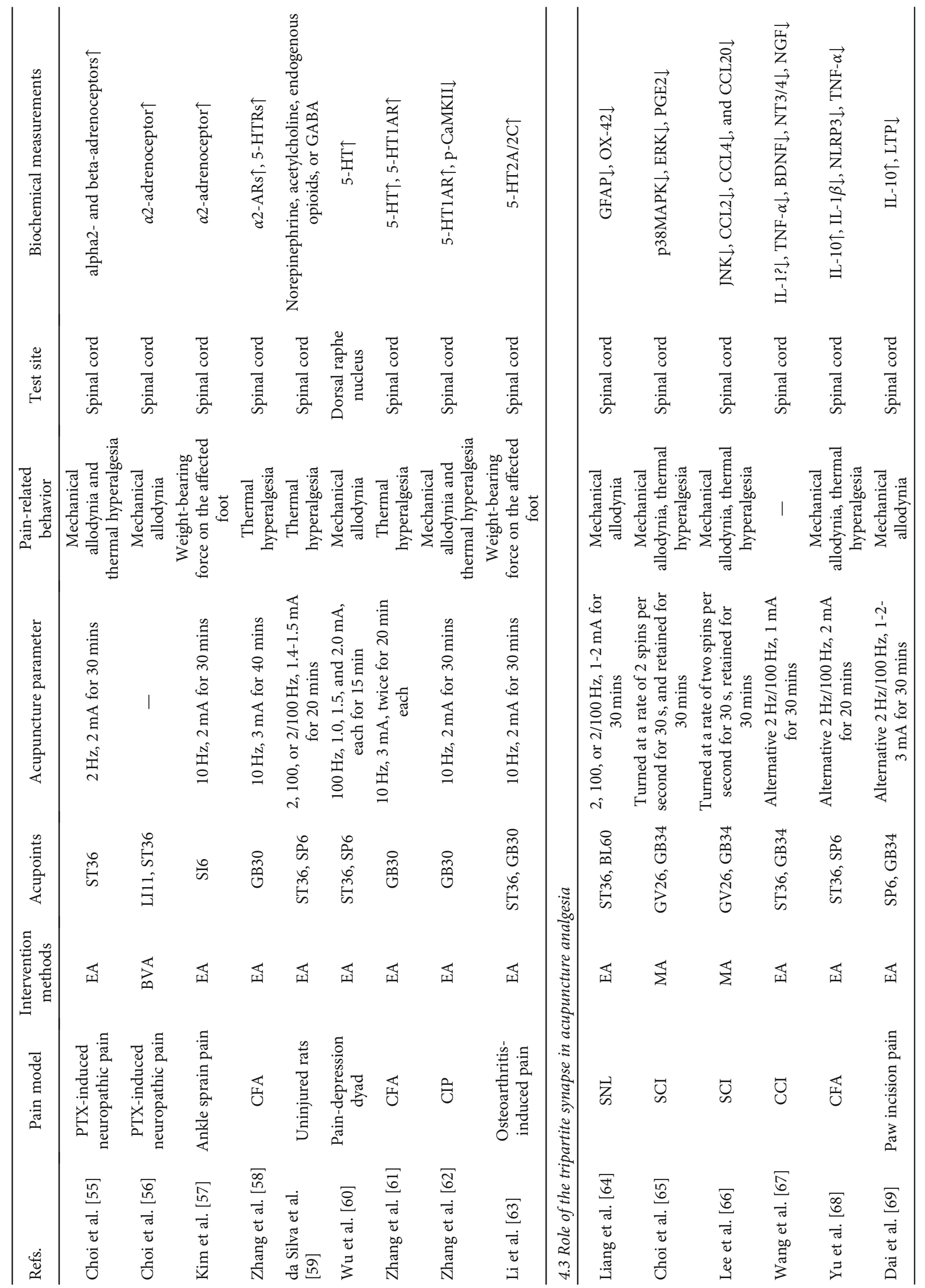




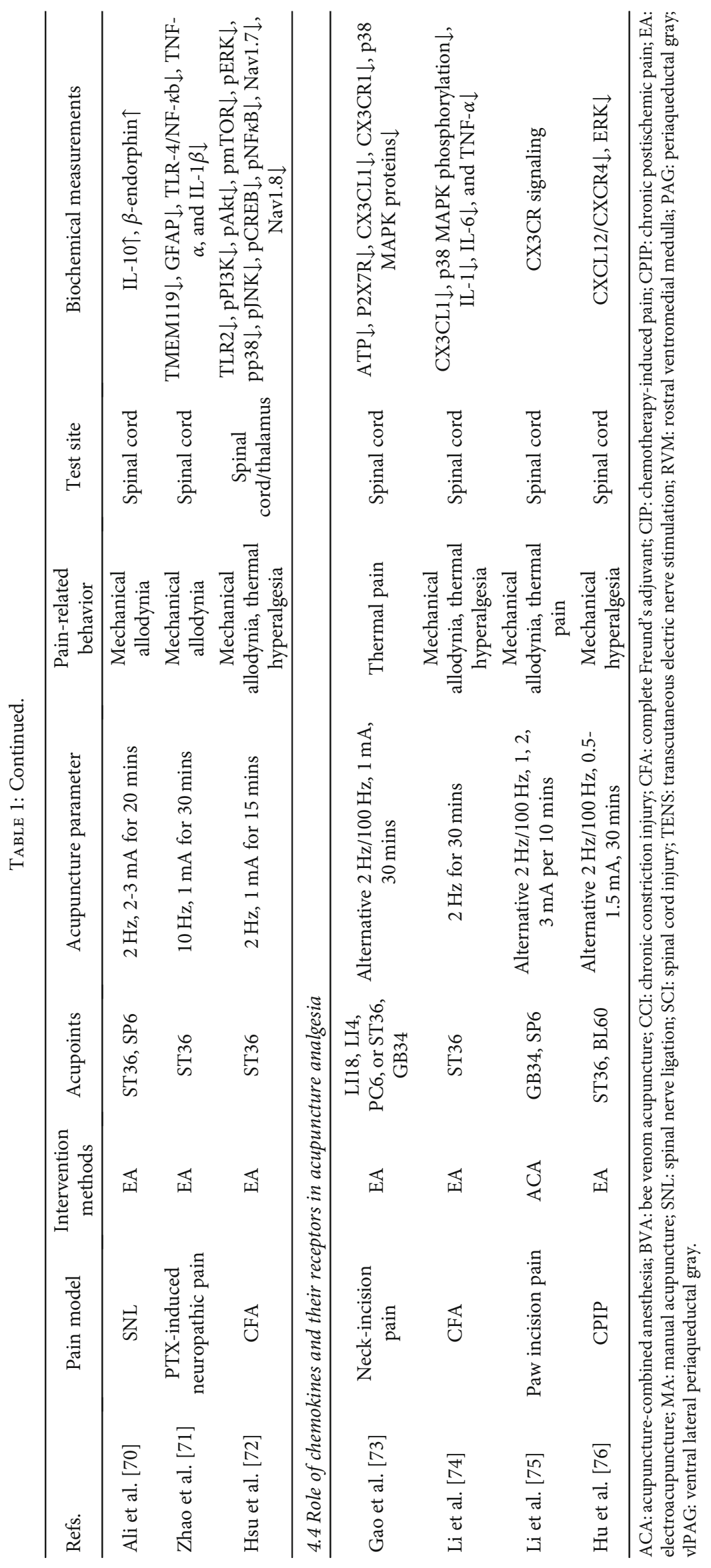


During this process, related neurons in the dorsal root ganglia depolarize and generate receptor potentials, converting physical or chemical information to electrical information. The electrical information is transmitted along the sensory pathway to the spinal cord and other primary centers. The integrated information from the primary center can be transmitted to higher centers in the brain to form the pain sensation, or to the motor neurons in the anterior horn to produce a reflex [20].

Pain sensitization is a remodeling mechanism of the central and peripheral nociceptive receptors. Continuous stimulation such as tissue and nerve injury remodels the nociceptor to produce pain sensitization, specifically, peripheral sensitization, is indicative of primary hyperalgesia, including peripheral nociceptive receptors and dorsal root ganglion DRG [21]. Neuroimmune interactions are essential for peripheral sensitization [22]. There are two types of peripheral inflammation: tissue and neurogenic inflammation. Tissue damage can cause the local release of histamine, vascular dilatation, increased blood flow, and vascular exudation, as well as the release of various cytokines/chemokines and agglutination factors. The immune cells (including macrophages, mast cells, neutrophils, and lymphocytes) release inflammatory mediators to aggravate pain and, at the same time, release endogenous opioid peptides to inhibit peripheral inflammatory pain [23]. In recent years, cytokines/chemokines have been found to be involved in regulating and sensitizing the excitability of nociceptors. Inflammatory cytokines (interleukin-1 $\beta$ (IL-1 $\beta$ ), IL-6 and tumor necrosis factor- $\alpha$ (TNF- $\alpha)$ ) mediate allodynia (pain resulting from a stimulus that would not normally provoke pain), hyperalgesia (increased response to noxious stimulation), and increasing the expression levels of substance $\mathrm{P}$ (SP) and prostaglandin E2 (PGE2) in neurons and glial cells in the DRG. Structurally, chemokines are similar to cytokines, and their receptors are expressed in the DRG. For instance, monocyte chemoattractant protein-1 (CCL2) and its receptor, $\mathrm{C}-\mathrm{C}$ chemokine receptor type 2 (CCR2), were both increased in the nerve injury model. Moreover, CCL2 can promote the interaction between immune cells and nociceptors, thus playing a key role in inflammatory and neuropathic pain [20]. At the same time, the excitatory effect of cytokines/chemokines on nociceptive neurons is manifested by regulating the opening of ion channels; specifically, the activation of chemokine receptors can regulate the current in voltage-gated calcium channels (VGCCs) [20].

Neurogenic inflammation is caused by chemicals, such as SP and calcitonin gene-related peptide (CGRP), that are released from primary nociceptive neurons [24]. Upon peripheral tissue injury, sustained nociceptive stimulation or inflammation sensitizes the nociceptors. These discharges activate ?-aminobutyric acid (GABA) neurons by acting on $N$-methyl-D-aspartate (NMDA) or non-NMDA receptors in the intermediate neurons of the spinal cord and cause depolarization by acting on adjacent primary afferent terminals. The discharge also produces a dorsal root reflex by retrograde conduction, leading to the resensitization of the injury receptors mediated by neurogenic inflammation and finally inducing paresthesia and allodynia [20]. In addition, the sympathetic nervous system participates in strengthening the neurogenic inflammatory process, and neuroimmune crosstalk plays an important role in peripheral sensitivity.

\section{Central Sensitization in Pain Transmission and Modulation Pathways Is a Crucial Target for Acupuncture Analgesia}

Central sensitization, namely secondary hyperalgesia, including the disbalance of the ascending excitatory pathway and descending pain modulatory system, plays a vital role in the development and maintenance of chronic pain [25]. In the ascending excitatory pathway, amplification of neural signaling within the central nervous system (CNS) produces a state of neuronal hyperactivity and hyperexcitability in the spinal cord and brain, leading to hyperalgesia [26]. This occurs mainly in the lamina I and lamina $\mathrm{V}$ neurons of the spinal cord, as well as in medullary reticular formation, thalamus, hypothalamus, cerebellum, amygdala, basal ganglia, hippocampus, cortexes S1 and S2, insula, anterior cingulate cortex (ACC), prefrontal cortex, and some related cortical areas in the parietal and temporal lobes [27-30]. The descending pain modulatory system arises from a number of supraspinal sites, including the midbrain periaqueductal gray (PAG) that projects indirectly to the spinal cord via the rostral ventromedial medulla (RVM), lateral and caudal dorsal reticular nucleus (DRN), and ventrolateral medulla, of which the PAG-RVM system has been most studied [31].

As shown in Table 1, the neuropathic pain models, including pain generated by chronic constriction injury, chemotherapy-induced pain, spinal nerve ligation, and spinal cord injury, have been widely used in the study of central sensitization-mediated acupuncture analgesia. Inflammatory pain induced by the administration of complete Freund's adjuvant also causes central sensitization, including the dual mechanisms of peripheral and central sensitization. Many of these studies have used electroacupuncture (EA) rather than manual acupuncture, and the acupressure points ST36 and SP6 have been most used. Both low frequencies at $2-15 \mathrm{~Hz}$ and, alternatively, higher frequencies between $2 \mathrm{~Hz}$ and $100 \mathrm{~Hz}$ were effective in EA analgesia, providing useful information for maximizing the effects of acupuncture analgesia in clinical settings.

4.1. Acupuncture Inhibits the Ascending Excitatory Pathway. As described in Section 1, nociceptive receptors are composed of types $\mathrm{A} \delta$ and $\mathrm{C}$ sensory fibers which transduce noxious stimuli from damaged peripheral tissues to the spinal cord. Neuroplasticity in the dorsal horn of the spinal cord assists and enhances pain perception by the nociceptive neurons. Central sensitization includes three processes: activation, sensitization, and modification of the CNS [32]. These are characterized by increases in the excitatory postsynaptic current (EPSC), including windup, long-term potentiation (LTP), and disinhibition. Among these, LTP caused by both high- and low-frequency electrical stimulation, nerve damage, or tissue damage is a process in which a transient synaptic activity can produce a long-lasting increase in synaptic strength. LTP relies on NMDA receptors (NMDARs) and voltage-dependent calcium channels, and 
results in calcium influx, thereby activating protein kinase A, protein kinase $\mathrm{C}$, and calcium/calmodulin-dependent protein kinase II (CaMKII) [33]. Protein synthesis is necessary for late-phase LTP (L-LTP), and administration of brainderived neurotrophic factor (BDNF) in the spinal dorsal horn and the hippocampus can induce L-LTP of C-fiber potentials [34]. Disinhibition includes a reduction of GABAergic and/or glycinergic inhibition and an enhancement of $\mathrm{A} \beta$ and $\mathrm{C}$ fiber sensitivity. The loss of inhibitory interneurons, impaired storage and/or release of inhibitory neurotransmitters, and impaired postsynaptic receptor activity have been proposed as the mechanisms underlying disinhibition [35]. Finally, the integrated signals travel through the spinal cord to the thalamus, where the location and intensity of the pain is processed. The signal then travels to the classic pain nerve circuit, the cerebral sensory cortex ( $\mathrm{S} 1$, S2), which distinguishes the location and duration of the pain [36]. Apart from S1 and S2, neuronal activities in the ACC, insular cortex, and amygdala also contribute to various aspects of pain perception, including the experience of discomfort [37].

In terms of the molecular mechanisms, primary nociceptive neurons release glutamate, SP, CGRP, adenosine triphosphate (ATP), and neurokinin-1 (NK1) into the spinal cord. These molecules interact with their receptors, including NMDARs, $\alpha$-amino-3-hydroxy-5-methyl-4-isoxazolepropionic acid receptors (AMPAR), metabotropic glutamate receptors (mGluR), SP receptors (NK1R), calcitonin receptor-like receptors (CRLR), and purinergic receptors (P2X, P2Y) of the spinal nociceptive projection neurons. This induces an influx of $\mathrm{Ca}^{2+}$ into the pain neurons of the spinal cord [38], activating calcium-dependent intracellular cascades, inducing NMDAR phosphorylation [39], ultimately resulting in the generation of nociceptive information throughout the spinothalamic pain pathway $[40,41]$. Thus, these mediators released by nociceptive neurons cause neural hyperexcitability and nociceptive transmission.

Glutamate is the most important and widely distributed excitatory neurotransmitter in the CNS and plays a critical role in the ascending excitatory pathway. Glutamate receptors are broadly divided into two groups, metabotropic glutamate receptors (mGluRs), and cation-permeable ionotropic glutamate receptors (iGluRs), which are, in turn, subdivided into NMDAR, Kainate-type iGluR, and AMPAR. Excitatory glutamatergic transmission plays a crucial role in the onset of chronic pain and is characterized by an increase in glutamate concentrations and receptor stimulation, leading to the transmission of pain messages [77, 78]. Somers et al. investigated the effects and potential mechanisms of transcutaneous electrical nerve stimulation (TENS) on neuropathic pain caused by chronic constriction injury (CCI). The mechanical allodynia was relieved by decreased concentrations of excitatory glutamate in the dorsal horn with a combination of low- and high-frequency TENS [79]. Recent evidence has shown that $2 \mathrm{~Hz}$ EA bilateral stimulation on the Zusanli (ST36) and Sanyinjiao (SP6) acupressure points produced analgesic effects through the downregulation of the $\mathrm{N}$ methyl-D-aspartate receptor type 2B (NR2B, NMDAR subunit), inhibiting the transmission of pain messages in a CCI rat model [42]. AMPAR consists of four subunits (GluR1R4). EA has been demonstrated to prevent phosphorylation of AMPAR, especially the GluR2 subunit, in a Complete Freund's Adjuvant (CFA) model $[43,44]$. In addition, the antinociceptive effect of EA is proposed to be related to the recovery of glutamate transporter (GT) expression, which can remove excess glutamate from the synaptic clefts. Both the spinal glutamate-aspartate transporter and GT1, which are mainly distributed in glial cells, were increased after EA treatment in CFA-injected rats as a result of proteasome downregulation [45]. A similar response has also been observed in a neuropathic pain model of spared nerve injury treated with EA [46]. Glutamatergic synaptic transmission is coupled with excess $\mathrm{Ca}^{2+}$ entry into projection neurons and results in the activation of the $\mathrm{Ca}^{2+}$-dependent enzyme $\mathrm{Ca}^{2+} /$ calmodulin-dependent protein kinase II (CamKII) and phosphorylation of the NR2B subunit of NMDAR at postsynaptic sites in the ACC, thus modulating visceral pain in a viscerally hypersensitive model [80]. It was reported that EA at ST36 and Kunlun (BL60) could reverse the actions of the calcium voltage-gated channel subunit and calcium voltage-gated channel auxiliary subunit $\gamma$, thus reducing chronic inflammatory pain (CIP) in the CFA rats [47].

4.2. Acupuncture Regulates the Descending Pain Modulatory System. It is now clear that the descending pain modulatory pathway can be both facilitatory and inhibitory, with a dynamic balance between the two functions. When acute pain turns to chronic pain, the descending facilitation function is dominant, leading to enhanced pain sensitization and even "mirror pain," in which the rostral RVM plays a key role. Descending projections from the RVM, identified as $\mathrm{ON}$ and OFF cells, facilitate and inhibit spinal nociceptive transmission, respectively. For example, RVM lesions or functional silencing can prevent pain sensitization induced by nerve injury. Excitatory amino acids, AMPAR, NMDAR, and the $\mathrm{BDNF} / \mathrm{TrkB}$ signaling pathway are also involved in the process of descending facilitation. Several neurotransmitters (e.g., opioid, GABA, norepinephrine, and 5hydroxytryptamine (5-HT)) are involved in these descending pathways [20]. It has been demonstrated that acupuncture functions mainly through facilitating the descending inhibitory system to ease pain, while the regulation of acupuncture on the descending facilitation system is poorly understood. Limited results have suggested that EA relieves inflammatory pain via inhibiting the activation of p38 mitogen-activated protein kinases (MAPK) in the central descending facilitation system [48]. In addition, EA potentiates the descending inhibitory control of 5-HT in the medulla via cannabinoid receptors on GABAergic but not glutamatergic neurons, thus inhibiting knee osteoarthritis pain [49].

4.2.1. Regulation of Opioids and Their Receptors by Acupuncture. Opioids and their receptors help reduce the excitatory transmitter release in the midbrain descending pathway, especially in the PAG-RVM system [81]. Kissiwaa et al. found that opioid receptors, mainly the $\mu$ opioid receptors (MORs), could inhibit glutamate release at synapses in 
the amygdala [82]. In addition, the analgesic capacity of MORs in the PAG was negatively regulated by glutamatebinding NMDAR NR1 subunits [83]. Until now, the activation of endogenous opioids (e.g., enkephalin, $\beta$-endorphin, and dynorphin)/opioid receptor (e.g., $\mu$ and $\delta$ opioid receptors) is the best-understood mechanism of acupuncture analgesia [50, 84-87]. Although different frequencies of EA can be reversed by the opioid antagonist naloxone, the types of opioids that mediate EA effects vary according to the EA frequency. For example, a single administration of $2 \mathrm{~Hz}$ EA at ST36 for 20 minutes can alleviate hyperalgesia during ethanol withdrawal through the mediation of MORs in the lateral habenula (LHb), an epithalamic structure rich in MORs. Activation of MORs may inhibit the release of glutamate in the LHb, which can block the descending nociceptive signal from the LHb to the PAG, thus reducing pain [50]. Besides MORs, $10 \mathrm{~Hz}$ EA at Huantiao (GB30) can significantly relieve paclitaxel-induced mechanical allodynia and hyperalgesia via the $\delta$ or $\kappa$ opioid receptors [51].

\subsubsection{Regulation of Nonopioid \\ Neurotransmitters by Acupuncture}

(1) GABAergic Inhibitory Interneuron Network. GABA is an important inhibitory neurotransmitter that is involved in the reduction of pain sensation through presynaptic inhibition [88]. The imbalance between excitatory glutamatergic and inhibitory GABAergic transmission, particularly decreased inhibition of GABAergic synaptic transmission in the spinal pain circuit, has been considered to underlie the development of central sensitization [23, 27, 52, 89, 90]. It was also found that RVM GABAergic neurons could facilitate mechanical pain by inhibiting dorsal horn enkephalinergic/GABAergic interneurons [91]. EA may initiate analgesia by increasing GABA expression on the descending pain modulatory pathways in the PAG [52]. Furthermore, the GABA receptor, a ligand-gated ionotropic receptor, is located mainly in postsynaptic neurons and contributes to the initiation of fast synaptic inhibition, thus being the major target for producing sedation [92]. EA at the bilateral L4 and L6 of Hua Tuo Jia Ji points (EX-B2) [53] or ST36 and Yanglingquan (GB34) points can significantly reduce neuropathic pain by increasing the GABA receptors in the spinal cord [54]. Moreover, EA can support the GABAergic system by reducing the rate of GABA reuptake. GABA transporter-1 (GAT1), the dominant neuronal GABA transporter, controls GABA concentrations, and EA was found to reduce GAT1 activity via activating the $\delta$-opioid receptor in the PAG [93]. Taken together, acupuncture can modulate the GABAergic system by increasing GABA expression, activating the GABA receptor, and inhibiting GABA reuptake.

(2) Norepinephrine. Norepinephrine is released from descending projection into the dorsal horn and helps to evoke an antinociceptive effect. The depletion of spinal norepinephrine caused mechanical hypersensitivity in a dosedependent manner in a chronic pain rat model [94]. Studies have clearly shown that norepinephrine is involved in acupuncture analgesia. A previous report by Choi et al. demon- strated that EA stimulation $(2 \mathrm{~mA}, 2 \mathrm{~Hz}, 30 \mathrm{~min}$, once every two days) at bilateral ST36 could significantly diminish paclitaxel-induced neuropathic pain. Further studies have shown that the antinociceptive effect of EA was mediated by spinal descending adrenergic pathways through the activation of the $\alpha 2$ - and $\beta$-spinal adrenoceptors in mice $[55,56]$. Consistent with these results, studies have shown that EA-induced analgesia in a rat model of an ankle sprain is mediated mainly by suppressing dorsal horn neuron activity through $\alpha$-adrenoceptors [57]. In addition, studies have shown that the analgesic effect of $10-\mathrm{Hz}-\mathrm{EA}$ was regulated by the spinal $\alpha 2 \mathrm{a}$-adrenoceptor, compared to the $\alpha 2 \mathrm{~b}$-adrenoceptor in a CFA-induced inflammatory pain model [58]. It has also been found that intrathecal injection of desipramine, a selective noradrenaline uptake inhibitor, increased the availability of spinal noradrenaline and prolonged the antinociception effect of EA [59].

(3) 5-Hydroxytryptamine. 5-HT (also called serotonin) arises largely from the RVM to the spinal cord and exerts biphasic modulation in the descending facilitation and inhibitory pathways. The facilitatory and inhibitory influence on the spinal processing of nociceptive information were mediated through recruitment of RVM ON or OFF cells, respectively, and depended on acute or chronic pain states and the type of receptor acted upon $[60,95]$. A low dose of 5-HT produces facilitation of fast EPSCs in the spinal cord, while a high dose of 5-HT induces inhibition of AMPA/kainite-receptor-mediated EPSCs $[88,96]$. Numerous studies have shown the involvement of the 5-HT and 5-HT receptors in acupuncture analgesia effects [60,97-99]. EA at $100 \mathrm{~Hz}$, but not 2, 50, or $2 / 100 \mathrm{~Hz}$, was effective in alleviating the pain-depression dyad and upregulating 5-HT in the DRN of reserpineinjected rats [60]. In a CFA-inflammatory pain model, $10 \mathrm{~Hz}$ EA inhibited thermal hyperalgesia through the activation of spinal 5-hydroxytryptamine 1A receptors (5-HT1AR) but not 5-HT2BR, 5-HT2CR, or 5-HT3R [58, 61]. Consistent with these results, EA activated spinal 5-HT1AR to alleviate both allodynia and hyperalgesia in CIP rats [62]. In an osteoarthritis model, the effectiveness and mechanisms of EA were focused on the involvement of spinal 5-HT2A/2C receptors [63]. Taken together, these data suggest that spinal 5-HT and its receptors are involved in acupuncture analgesia but the effects differ according to both animal models and EA frequencies.

\subsection{Role of the Tripartite Synapse in Acupuncture Analgesia}

4.3.1. Neuroglial Crosstalk Regulates the Neural Plasticity. Vladimir Parpura first proposed the concept of the "tripartite synapse" in 1994 [100]. This concept added glial cells (astrocytes and microglia) to the original classic pre- and postsynaptic neuronal cells, thus emphasizing the important roles of glial cells in synaptic transmission and regulation. It is noteworthy that recent studies have shown that central sensitization driven by neuroinflammation in the CNS is accompanied by glial activation, including the activation of astrocytes, microglia, and oligodendrocytes in the spinal cord and brain [101]. Lau and coworkers found that 
L5 spinal nerve ligation (SNL) caused the upregulation of cyclooxygenase-1 (COX-1) and COX-2 in spinal microglial and neuronal cells; these enzymes are involved in the production of prostaglandins (PGs) via arachidonic acid pathways and, in turn, lead to the development of neuropathic pain [102]. Functional imaging also showed elevated cerebral levels of the translocator protein, a marker of glial activation, in patients with chronic low back pain [103]. Therefore, chronic pain may also be the result of "gliopathy" [104].

It is increasingly clear that neural plasticity is regulated by neuroglial crosstalk. After peripheral tissue or nerve damage, neurotransmitters such as glutamate, SP, and CGRP released from nociceptive primary afferent fibers in the dorsal horn not only cause intense high-frequency activation of the neural postsynaptic receptors and amplification of the postsynaptic current but also interact with their corresponding receptors on microglia and astrocytes [105-107], activating the voltage-dependent calcium channel and inducing calcium entry into the neuron. Glia-derived substances are termed gliotransmitters and include cytokines (IL-1, IL-6, and TNF$\alpha$ ), chemokines (CCL2 [108] and CXCL1 [109]), and inflammatory mediators (e.g., bradykinin, PGs, and nitric oxide). These chemicals promote an inflammatory environment [101] and act as chemical mediators to amplify neuroglial reactivity in a paracrine manner, favoring the elevation of these mediators in the dorsal horn of the spinal cord [110]. As an example, TNF- $\alpha$ acting at the TNF- $\alpha$ receptor 1 (TNFR1) functions in the development of pain by facilitating excitatory synaptic signaling in the acute phases after nerve injury in CCI mice compared with sham control mice [111]. TNF- $\alpha$ contributes to neural plasticity through mechanisms involving the downregulation of GTs, upregulation of the glutamate concentration in the synapse, and phosphorylation of NMDAR. TNF- $\alpha$ subsequently promotes the facilitation of excitatory synaptic transmission and downregulates the expression of GABA receptors, thus reducing the inhibition of excitatory transmission [112]. Chemokines can also regulate the interactions of neurons and glial cells, e.g., CCL2 derived from astrocytes can "talk to" neurons by regulating neuronal activity via c-Jun-N-terminal kinase (JNK) MAPK [108]. These mediators produced by active glial cells contribute to neural plasticity. During the process, the activated glia can release prostaglandin, BDNF, nitric oxide, and other neuroactive substances; reduce the inhibitory effects of GABA; and upregulate the expression of NMDARs, thereby increasing nerve excitability and maintaining neuropathic pain $[22,113]$. Moreover, modification of glial cell numbers in the brain is likely correlated with the emotional experience of pain, as well as mood disorders such as depression and anxiety [114]. Once activated, the glial cells in the spinal cord release cytokines that provide positive feedback, further enhancing excitatory synaptic transmission.

4.3.2. Inhibition of Glial Activity by Acupuncture. It has been shown that in the neuropathic pain model induced by SNL, the inhibition of spinal microglia and astrocytes mediates the immediate and long-term EA analgesia, respectively [64]. It has also been reported that the analgesic efficacy of
EA might be related to the modulation of microglial and astrocyte activation [115-117]. Acupuncture has been reported to suppress signal transduction pathways and key molecules, including p38 MAPK, extracellular regulated kinases (ERK), and JNK, in microglial and astrocyte activation in pain processing $[65,66,118-120]$. Previous results observed that the acupuncture analgesic effect is related to spinal cytokines and neurotrophic factors released by glial cells $[67,121,122]$. Repeated EA treatment at the bilateral ST36 and GB34 points once a day can relieve chronic pain and suppress the elevated mRNA expression of TNF $\alpha$ and IL- $1 \beta$ in the spinal cord of CCI rats [67]. EA $(2 / 100 \mathrm{~Hz}$, $2 \mathrm{~mA}$ ) for five consecutive days can significantly increase the mechanical threshold and thermal latency after CFA injection. This could be partially associated with the suppression of proinflammatory cytokines (e.g., TNF- $\alpha$ and IL-1 $\beta$ ) and the stimulation of IL-10 in the spinal cord. IL-10, produced by the spinal cord, is the key anti-inflammatory cytokine for relieving both inflammatory pain $[68,69]$ and neuropathic pain [70]. Paclitaxel significantly activates both microglia and astrocytes and increases the expression of inflammatory cytokines (IL- $1 \beta$ and TNF- $\alpha$ ) in the lumbar spinal cord. EA treatment $(10 \mathrm{~Hz}, 1 \mathrm{~mA})$ at the bilateral ST36 point in rats suppressed the expression of inflammatory cytokines through the downregulation of the TLR4/NF- $\kappa \mathrm{B}$ pathway as well as suppressing activated microglia and astrocytes [71]. Besides TLR4, increased expression of TLR2 in the spinal cord and thalamus was also reportedly suppressed by EA in a CFA model [72]. Therefore, the downregulation of gliotransmitters by acupuncture can prevent the activation of neuroglial crosstalk, thus contributing to the easing of chronic pain. However, the evidence on how acupuncture modulates glial cells to inhibit excitatory synaptic transmission is still incomplete.

\subsection{Role of Chemokines and Their Receptors in Acupuncture Analgesia}

4.4.1. Introduction to the Chemokine System. Chemokines, 8$12 \mathrm{kDa}$ secreted proteins, constitute the largest family of cytokines. According to the number and spacing of cysteines, chemokines consist of two major families, $\mathrm{CC}\left(\mathrm{CC}_{1-28}\right)$ and $\mathrm{CXC}\left(\mathrm{CXC}_{1-16}\right)$ chemokines, as well as two minor families, $\mathrm{XC}\left(\mathrm{XC}_{1-2}\right)$ and $\mathrm{CX} 3 \mathrm{C}(\mathrm{CX} 3 \mathrm{CL} 1)$ chemokines [123]. All chemokines bind to the members of a family of seven transmembrane-spanning heterotrimeric $\mathrm{G}$ protein-coupled receptors (GPCRs). Chemokines are regarded as important mediators of inflammation and help to control the positioning and migratory patterns of immune cells. Immune cell residence in primary ( $\mathrm{T}$ cells, B cells), secondary (lymph nodes, spleen, Peyer's patches), and tertiary lymphoid organs are under the fine control of this complex system of approximately 50 endogenous chemokines [123]. The development of $\mathrm{T}$ cells in the thymus depends on the interaction of epithelial-derived CCL21, CCL25, and CXCL12 with CCR7, CCR9, and CXCR4, respectively, expressed on T cell progenitors [124]. In contrast to the thymus, the homeostasis and development of immune cells in the bone marrow seems to be governed by the opposing forces of interactions between 
CXCL12/CXCR4 and CCL2/CCR2 [123]. Chemokines, considered as regulators of peripheral immune cell transport, are capable of inducing the migration of $\mathrm{T}$, NK cells, dendritic cells, and/or macrophages [125]. There has been growing recognition that the chemokine system orchestrates immune cell migration (e.g., macrophages [126] and lymphocytes [127]) into the DRG and CNS. This plays a critical role in the central sensitization in the early initiation of pain perception [128]. Besides, chemokines and their receptors expressed by neurons and glial cells in the CNS have been shown to mediate neuroglial communication and nociceptive signal transmission at different anatomical locations, including nerves, the DRG, spinal cord, and brain $[129,130]$.

\subsubsection{Acupuncture Analgesia via Regulation Chemokine and} Their Receptors. Concomitant with the increasing use of acupuncture to alleviate pain, attention has been paid in recent years to the mechanism of acupuncture analgesia from the perspective of chemokines.

(1) CX3CL1/CX3CR1. CX3CL1 is specifically expressed in neurons and binds to the CX3CR1 receptor on the microglial cell membrane [125], activating the MAPK pathway. MAPKs play important roles in information transmission between neurons and glial cells as well as the genesis of pain hypersensitivity induced by CX3CL1/CX3CR1 [131, 132]. Gao et al. reported that peripheral injury to the primary afferent nociceptive neurons caused the release of CX3CL1 into the spinal cord, which activated the production of $\mathrm{TNF} \alpha$ in a p38 MAPK-dependent mechanism in microglia. In turn, the activation of TNF- $\alpha$ regulates CCL2 expression in astrocytes in a JNK MAPK-dependent manner. CCL2 subsequently activates central neurons through CCR2, eventually leading to neuropathic pain [133]. Neutralizing antibodies to CX3CL1 or CX3CR1 could attenuate mechanical hyperalgesia in neuropathic pain models $[134,135]$. It has been demonstrated that neuron-microglia interactions are mediated by purinergic receptors, and, subsequently, by CX3CL1/CX3CR1. The purinergic P2X7R/CX3CL1/CX3CR1 pathway, following intracellular phosphorylation of microglial p38 MAPK [136] which subsequently stimulates the release of IL-6 and IL-1 $\beta$ [137], plays a key role in nociceptive signal transmission [138].

In a rat neck-incision pain model, Gao et al. found that two sessions of EA at Futu (LI18), Hegu (LI4), Neiguan (PC6), or ST36, GB34 could significantly relieve thermal pain, followed by the downregulation of ATP/P2X7R/ CX3CL1/CX3CR1 signaling and suppression of its downstream p38 MAPK pathway in the upper cervical spinal cord after three sessions. Thereby, EA suppressed ATP/P2X7R/ CX3CL1/CX3CR1/p38 MAPK-induced neuroglial crosstalk in pain processing [73]. The results are consistent with the reports by $\mathrm{Li}$ that $2 \mathrm{~Hz}$ EA at ST36 for 30 mins reduced the overexpression of CX3CL1 other than CX3CR1 in the spinal cord of the CFA model rats. EA inhibited the activation of neuronal and microglial cells and decreased p38 MAPK signaling and the downstream proinflammatory cytokines IL-1, IL-6, and TNF- $\alpha$. They also found that EA did not inhibit the expression of p38 MAPK but inhibited its phosphorylation [74]. Slightly different from their results, the report by Li et al. [75] demonstrated that mechanical allodynia and thermal pain induced hyperalgesia by paw incision was significantly suppressed by acupuncture-combined anesthesia (ACA). However, the analgesic effect of ACA was not apparent in CX3CR1 knockout mice and was also blocked when a neutralizing antibody to CX3CR1 was intrathecally injected $1 \mathrm{~h}$ before ACA in C57BL/6J mice, suggesting that CX3CR1 in microglia is not only involved in postincision pain, but also in ACA-induced analgesia.

(2) CXCL12/CXCR4. CXCL12 and CXCR4 are, respectively, expressed in neurons and glial cells in the central nervous system [139], and the CXCL12/CXCR4 activation leads to increased pain sensitivity in the spinal cord. A study by Luo et al. demonstrated that CXCL12 and CXCR4 are upregulated in the spinal cord dorsal horn in chronic postischemic pain (CPIP) mice. Intrathecal blocking of CXCR4 improved mechanical allodynia, suggesting an important role of spinal CXCL12/CXCR4 signaling in ameliorating the pain response [140]. Hu et al. found that EA exerted an analgesic effect on the same rat model of CPIP by suppressing the overexpression of CXCL12/CXCR4 in the spinal cord dorsal horn. Furthermore, they found that EA can effectively inhibit excessive activation of glial cells in the spinal cord and markedly reduce downstream ERK pathway activation, thus reducing the central sensitization and exerting an analgesic effect [76].

(3) CCL2/CCR2. CCL2 is expressed in spinal astrocytes and induces neuronal activation via CCR2 to increase excitatory synaptic transmission (astrocyte-to-neuron signaling), contributing to central sensitization and neuropathic pain development. It has been shown that CCL2/CCR2 was upregulated in the spinal cord via the JNK pathway after SNL [108]. CCL2 can also rapidly increase NMDA-induced current and spontaneous EPSCs [108] or inhibit GABAinduced currents [141] in dorsal horn neurons, all of which are critically involved in the maintenance of pain. Furthermore, spinal administration of CCL2 induced thermal hyperalgesia via activating the spinal transient receptor potential vanilloid 1 (TRPV1) receptors [142]. Lee et al. demonstrated acupuncture at Shuigou (GV26) and GB34 significantly alleviated both mechanical allodynia and thermal hyperalgesia after spinal cord injury at L4-L5. It is noteworthy that acupuncture inhibited the astrocyte expression of CCL2, which is known to be mediated through the JNK pathway and contributes to excitatory synaptic transmission. Apart from the findings on CCL2, Lee also showed that JNK-dependent CCL4 and CCL20 expression was significantly decreased by acupuncture treatment [66].

(4) CXCL1/CXCR2. CXCL1 and CXCR2 are expressed in astrocytes and neurons, respectively, in the spinal cord, and CXCL1/CXCR2 in the lumbar spinal cord has been demonstrated to play key roles in pain processing. The application of CXCL1 in the spinal cord acted on CXCR2, inducing the expression of phosphorylated ERK and cAMP-response element-binding protein, c-fos, and COX- 2 in the spinal cord 


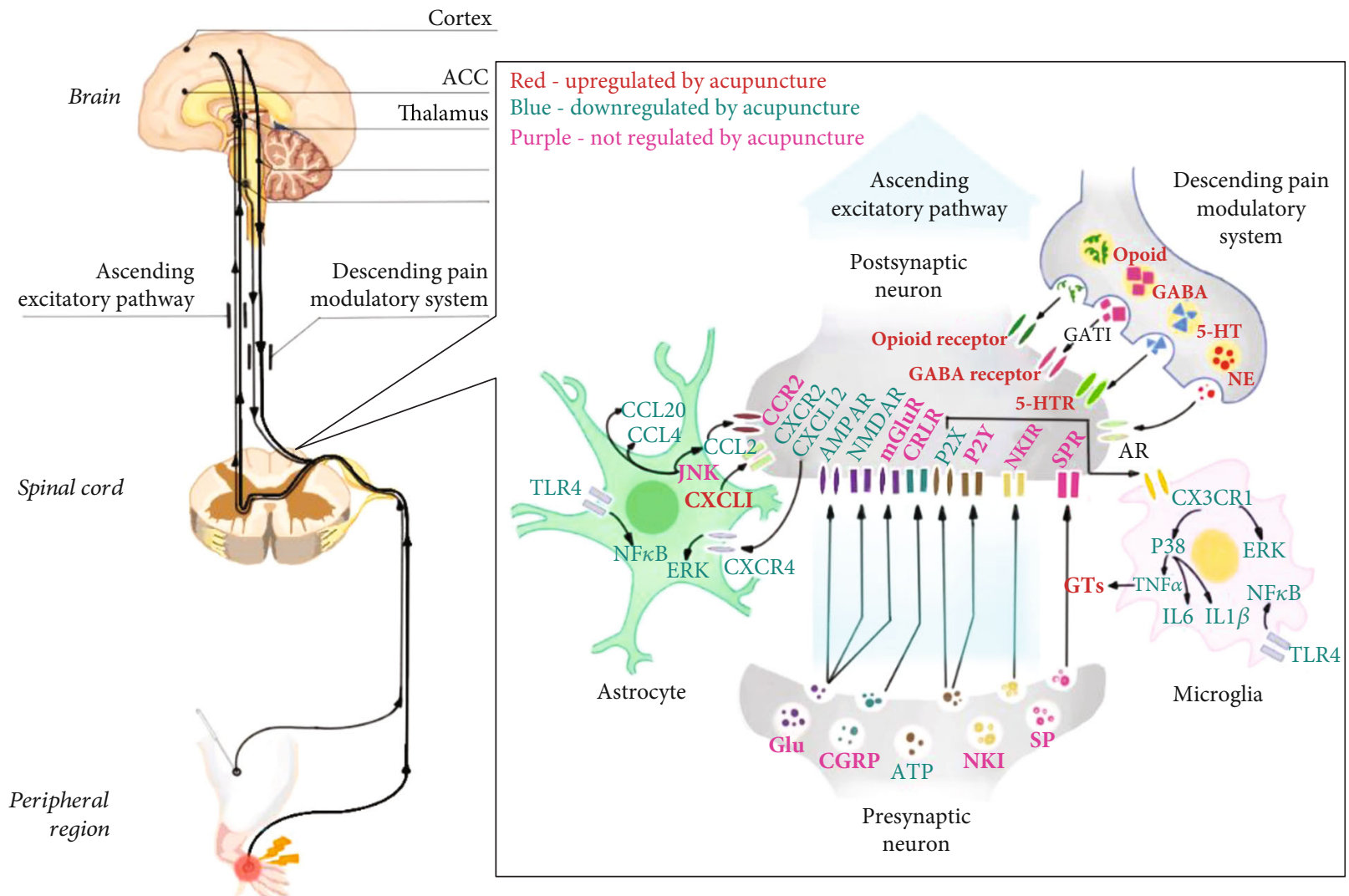

FIgURE 2: Role of neuroglial crosstalk and synaptic plasticity mediated central sensitization in acupuncture analgesia. The titles of neurotransmitters, neuropeptides, and immune factors are presented in red, blue, and purple, as the figure shows. Factors in red are upregulated by acupuncture, while factors in blue are downregulated by acupuncture. Factors in purple are activated or inhibited in the central sensitization process but are not regulated by acupuncture. ACC: anterior cingulate cortex; PAG: periaqueductal gray; RVM: rostral ventromedial medulla; GABA: ?-aminobutyric acid; 5-HT: 5-hydroxytryptamine; 5-HTR: 5-hydroxytryptamine receptor; NE: norepinephrine; AR: adrenergic receptor; GAT1: GABA transporter 1; Glu: glutamate; AMPAR: $\alpha$-amino-3-hydroxy-5-methyl-4isoxazolepropionic acid receptor; NMDAR: $N$-methyl-D-aspartate receptor; mGluR: metabotropic glutamate receptors; CGRP: calcitonin gene-related peptide; CRLR: calcitonin receptor-like receptor; ATP: adenosine triphosphate; P2X: P2X receptor; P2Y: P2Y receptor; NK1: neurokinin-1; NK1 receptor: neurokinin-1 receptor; SP: substance P; SPR: substance P receptor; CX3CL1: C-X3-C motif chemokine ligand 1; CX3CR1: C-X3-C chemokine receptor 1; ERK: extracellular signal-regulated kinases; TNF- $\alpha$ : tumor necrosis factor- $\alpha$; IL-6: interleukin6; IL-1 $\beta$ : interleukin-1 $\beta$; GT: glutamate transporter; TLR4: toll-like receptor 4; JNK: c-Jun-N-terminal kinase; CCL2: C-C motif chemokine ligand 2; CCL4: C-C motif chemokine ligand 4; CCL20: C-C motif chemokine ligand 20; CCR2: C-C chemokine receptor 2; CXCL1: C-X-C motif chemokine ligand 1; CXCR2: C-X-C chemokine receptor 2; CXCL12: C-X-C motif chemokine ligand 12; CXCR4: C$\mathrm{X}-\mathrm{C}$ chemokine receptor 4 . Red, promoted by acupuncture; blue, inhibited by acupuncture.

neurons and leading to the subsequent maintenance of neuropathic pain [109]. Similarly, Cao and coworkers showed that astrocytes were activated after inflammation and released CXCL1 in the spinal cord, which could then act on CXCR2 to induce ERK activation, synaptic transmission, and COX-2 expression in dorsal horn neurons, ultimately contributing to the pathogenesis of CFA-induced inflammatory pain [143]. In addition, Xu et al. found that astrocyte-secreted CXCL1 activated spinal cord dorsal horn neurons to express CXCR2 in cancer pain models [144]. Taken together, CXCL1/CXCR2 is involved in the development of neuropathic, inflammatory, and cancer pain.

However, it has also been demonstrated that CXCL1/CXCR2 is involved in pain relief. Previously, a study by $\mathrm{Cao}$ et al. demonstrated intrathecal administration of recombinant CXCL1 in wild-type mice significantly reduced spinal nerve L5 transection- (L5Tx-) induced mechanical hypersensitivity. Due to CXCL1's chemotaxis, it is capable of associating with the increased number of opioid peptides produced by infiltrating neutrophils in the lumbar spinal cord [145]. Similarly, Guo and his colleagues showed that bone marrow stromal cell-induced monocytes secrete CXCL1 which could cross the blood-brain barrier and contribute to pain relief. The CXCL1/CXCR2 signaling triggers opioid release by activation of central $\mu$-opioid receptors in the RVM [146]. Furthermore, we have observed a novel function of acupuncture-derived CXCL1 in the CFA rat model. In our unpublished data, it was found that acupuncture at ST36 could induce high CXCL1 levels in CFA rat serum. CXCL1 neutralizing antibodies reduced the acupuncture analgesic effect by $20 \%$. Our results demonstrated that acupuncturederived CXCL1 can induce spinal cord CXCR2 desensitization, blocking COX2 production in the spinal cord. These 
phenomena showed that the same chemokines involved in pain sensitization may also be neuroprotective and promote pain relief under certain conditions.

\section{Conclusion}

To conclude (as shown in Figure 2), it is well established that pain is a hypersensitivity state caused by peripheral and central sensitization. Central sensitization is modulated by the ascending excitatory pathway and the descending pain modulatory system. With the emergence of the "tripartite synapse" concept, neuroglial cells are considered as active partners of neurons at the synapse and can contribute to central sensitization. It is increasingly appreciated that neurotransmitters (e.g., glutamate, opioid, GABA, norepinephrine, and 5-HT), inflammatory cytokines, and chemokines are implicated in this crosstalk. Acupuncture analgesia involves the ascending excitatory pathway and the descending pain modulatory system through the downregulation of glutamate and upregulation of opioids, GABA, norepinephrine, and 5-HT. Evidence indicates that the occurrence and maintenance of pain are closely related to immune responses, and the role of cytokines/chemokines in mediating neuroglial communication has attracted much attention in recent years. Acupuncture analgesia has also been demonstrated to inhibit cytokines such as IL- $1 \beta$, IL-6, and TNF- $\alpha$ and upregulate IL-10, as well as modulating chemokines and their receptors such as CX3CL1/CX3CR1, CXCL12/CXCR4, CCL2/CCR2, and CXCL1/CXCR2. Furthermore, acupuncture has been found to regulate downstream neural MAPK signaling (e.g., p38, ERK, and JNK pathways), which contribute to the activation of nociceptive neurons. However, the responses of chemokines to acupuncture differ between pain model types, acupuncture methods, and parameters, requiring future clarification of the exact mechanisms. Taken together, the inhibition of neuroglial plasticitymediated central sensitization is one of the critical mechanisms in acupuncture analgesia, contributing to the wider application of acupuncture, alone or in combination with pain medication, in the enhancement of treatment effectiveness and the lowering of pain medication dosages and decreasing the risk of debilitating adverse effects. The acupuncture parameters described in these studies, particularly those of EA stimulation, also provide significant information for maximizing the effect of acupuncture in the clinical setting.

\section{Abbreviations}

$\begin{array}{ll}\text { RTCs: } & \text { Randomized controlled trials } \\ \text { IL-1 } \beta \text { : } & \text { Interleukin-1 } \beta \\ \text { TNF- } \alpha: & \text { Tumor necrosis factor- } \alpha \\ \text { SP: } & \text { Substance P } \\ \text { PGE2: } & \text { Prostaglandin E2 } \\ \text { DRG: } & \text { Dorsal root ganglion } \\ \text { VGCCs: } & \text { Voltage-gated calcium channels } \\ \text { CGRP: } & \text { Calcitonin gene-related peptide } \\ \text { GABA: } & \text { ?-Aminobutyric acid } \\ \text { NMDA: } & \text { N-methyl-D-aspartate }\end{array}$

CNS: Central nervous system

EPSC: $\quad$ Excitatory postsynaptic current

LTP: Long-term potentiation

NMDARs: N-methyl-D-aspartate receptors

CaMKII: Calcium/calmodulin-dependent protein kinase II

L-LTP: $\quad$ Late-phase LTP

BDNF: Brain-derived neurotrophic factor

ACC: Anterior cingulate cortex

ATP: Adenosine triphosphate

NK1: $\quad$ Neurokinin-1

AMPAR: $\quad \alpha$-Amino-3-hydroxy-5-methyl-4-isoxazolepropionic acid receptors

mGluR: Metabotropic glutamate receptors

SPR: $\quad$ SP receptor

CRLR: Like receptor

NK1R: NK1 receptor

iGluR: $\quad \alpha$-Amino-3-hydroxy-5-methyl-4-isoxazolepropionic acid receptor, ionotropic glutamate receptors

TENS: Transcutaneous electrical nerve stimulation

CCI: Chronic constriction injury

EA: Electroacupuncture

NR2B: N-methyl-D-aspartate receptor type 2B

CFA: Complete Freund's adjuvant

GT: Glutamate transporter

CIP: $\quad$ Chronic inflammatory pain

PAG: $\quad$ Periaqueductal gray

RVM: Rostral ventromedial medulla

DRN: Dorsal reticular nucleus

5-HT: 5-Hydroxytryptamine

MOR: $\quad \mu$ opioid receptor

LHb: Lateral habenula

GAT1: $\quad$ GABA transporter 1

5-HT1AR: 5-Hydroxytryptamine $1 \mathrm{~A}$ receptors

SNL: $\quad$ Spinal nerve ligation

COX-1: Cyclooxygenase-1

PGs: Prostaglandins

TNFR1: $\quad$ TNF- $\alpha$ receptor 1

MAPK: Mitogen-activated protein kinase

ERK: $\quad$ Extracellular signal-regulated kinase

MA: $\quad$ Manual acupuncture

GPCRs: G protein-coupled receptors

JNK: c-Jun-N-terminal kinase

ACA: Acupuncture-combined anesthesia

CPIP: $\quad$ Chronic postischemic pain

TRPV1: Transient receptor potential vanilloid 1

L5Tx: L5 transection

\section{Data Availability}

The generated or analyzed data used to support the findings of this study are included within the article.

\section{Conflicts of Interest}

The authors have no conflict of interest regarding this paper. 


\section{Authors' Contributions}

The authors contributed in the following manner: Zhongxi Lyu and Yongming Guo contributed to the concept design, data collection, and paper writing; Wen Fan and Baomin Dou contributed to the figures and graphic abstract edit; Yinan Gong contributed to the data collection and analysis; Ningcen $\mathrm{Li}$, Shenjun Wang, Yuan $\mathrm{Xu}$, Yangyang Liu, Bo Chen, and Yi Guo contributed to the language modification and text check; and Xiaowei Lin and Zhifang Xu contributed to the concept design and paper review. Zhongxi Lyu and Yongming Guo contributed equally to this work.

\section{Acknowledgments}

This study was financially supported by the National Key R\&D Program of China No. 2019YFC1709003; the National Natural Science Foundation of China (NSFC) No. 81704146, 81873369, and 81873368; the Natural Science Foundation of Tianjin No. 20JCQNJC00280.

\section{References}

[1] L. J. Crofford, "Chronic pain: where the body meets the brain," Transactions of the American Clinical and Climatological Association, vol. 126, pp. 167-183, 2015.

[2] A. Vincent, M. O. Whipple, S. J. McAllister, K. M. Aleman, and J. L. St Sauver, "A cross-sectional assessment of the prevalence of multiple chronic conditions and medication use in a sample of community-dwelling adults with fibromyalgia in Olmsted County, Minnesota," BMJ Open, vol. 5, no. 3, article e006681, 2015.

[3] P. Baral, S. Udit, and I. M. Chiu, "Pain and immunity: implications for host defence," Nature Reviews Immunology, vol. 19, no. 7, pp. 433-447, 2019.

[4] B. I. Martin, R. A. Deyo, S. K. Mirza et al., "Expenditures and health status among adults with back and neck problems," JAMA, vol. 299, no. 6, pp. 656-664, 2008.

[5] M. Verret, F. Lauzier, R. Zarychanski et al., "Perioperative use of gabapentinoids for the management of postoperative acute pain: protocol of a systematic review and meta-analysis," Systematic Reviews, vol. 8, no. 1, p. 24, 2019.

[6] N. B. Finnerup, "Nonnarcotic methods of pain management," The New England Journal of Medicine, vol. 380, no. 25, pp. 2440-2448, 2019.

[7] K. Kingwell, "Screening for cleaner pain relief," Nature Reviews. Drug Discovery, vol. 15, no. 10, p. 677, 2016.

[8] NIN Consensus Development Panel on Acupuncture, "Acupuncture," JAMA: The Journal of the American Medical Association, vol. 280, no. 17, pp. 1518-1524, 1998.

[9] Q. Zhang, A. Sharan, S. A. Espinosa, D. Gallego-Perez, and J. Weeks, "The path toward integration of traditional and complementary medicine into health systems globally: the World Health Organization report on the implementation of the 2014-2023 strategy," Journal of Alternative and Complementary Medicine, vol. 25, no. 9, pp. 869-871, 2019.

[10] M. Abou el Hassan, K. Huang, M. B. K. Eswara et al., "Cancer cells hijack PRC2 to modify multiple cytokine pathways," PLoS One, vol. 10, no. 6, article e0126466, 2015.

[11] Y. Guo, H. Zhao, F. Wang et al., "Recommendations for acupuncture in clinical practice guidelines of the national guide- line clearinghouse," Chinese Journal of Integrative Medicine, vol. 23, no. 11, pp. 864-870, 2017.

[12] A. J. Vickers, E. A. Vertosick, G. Lewith et al., "Acupuncture for Chronic Pain: Update of an Individual Patient Data MetaAnalysis," The Journal of Pain, vol. 19, no. 5, pp. 455-474, 2018.

[13] L. Zhao, J. Chen, Y. Li et al., "The long-term effect of acupuncture for migraine prophylaxis," JAMA Internal Medicine, vol. 177, no. 4, pp. 508-515, 2017.

[14] E. Deval, J. Noel, N. Lay et al., "ASIC3, a sensor of acidic and primary inflammatory pain," The EMBO Journal, vol. 27, no. 22, pp. 3047-3055, 2008.

[15] S. Wang, Y. Dai, K. Kobayashi et al., "Potentiation of the P2X3 ATP receptor by PAR-2 in rat dorsal root ganglia neurons, through protein kinase-dependent mechanisms, contributes to inflammatory pain," The European Journal of Neuroscience, vol. 36, no. 3, pp. 2293-2301, 2012.

[16] R. L. Silva, A. H. Lopes, R. M. Guimaraes, and T. M. Cunha, "CXCL1/CXCR2 signaling in pathological pain: role in peripheral and central sensitization," Neurobiology of Disease, vol. 105, pp. 109-116, 2017.

[17] R. R. Ji, Z. Z. Xu, and Y. J. Gao, "Emerging targets in neuroinflammation-driven chronic pain," Nature Reviews Drug Discovery, vol. 13, no. 7, pp. 533-548, 2014.

[18] F. A. Pinho-Ribeiro, W. A. Verri Jr., and I. M. Chiu, "Nociceptor sensory neuron-immune interactions in pain and inflammation," Trends in Immunology, vol. 38, no. 1, pp. 5-19, 2017.

[19] S. Talbot, S. L. Foster, and C. J. Woolf, "Neuroimmunity: physiology and pathology," Annual Review of Immunology, vol. 34, no. 1, pp. 421-447, 2016.

[20] J. H. Jun Chen, B. Fan, S. Yu, and J. Wang, "The underlying mechanisms of chronic pain: from basic to clinical," Chinese Journal of Pain Medicine, vol. 20, no. 2, pp. 70-80, 2014.

[21] C. J. Woolf and Q. Ma, "Nociceptors-noxious stimulus detectors," Neuron, vol. 55, no. 3, pp. 353-364, 2007.

[22] K. Ren and R. Dubner, "Interactions between the immune and nervous systems in pain," Nature Medicine, vol. 16, no. 11, pp. 1267-1276, 2010.

[23] A. I. Basbaum, D. M. Bautista, G. Scherrer, and D. Julius, "Cellular and molecular mechanisms of pain," Cell, vol. 139, no. 2, pp. 267-284, 2009.

[24] W. D. Willis Jr., "Dorsal root potentials and dorsal root reflexes: a double-edged sword," Experimental Brain Research, vol. 124, no. 4, pp. 395-421, 1999.

[25] P. Boadas-Vaello, S. Castany, J. Homs, B. Alvarez-Perez, M. Deulofeu, and E. Verdu, "Neuroplasticity of ascending and descending pathways after somatosensory system injury: reviewing knowledge to identify neuropathic pain therapeutic targets," Spinal Cord, vol. 54, no. 5, pp. 330-340, 2016.

[26] C. J. Woolf, "Central sensitization: implications for the diagnosis and treatment of pain," Pain, vol. 152, Supplement, pp. S2-15, 2011.

[27] A. Latremoliere and C. J. Woolf, "Central sensitization: a generator of pain hypersensitivity by central neural plasticity," The Journal of Pain, vol. 10, no. 9, pp. 895-926, 2009.

[28] X. M. Shao, J. Sun, Y. L. Jiang et al., "Inhibition of the CAMP/PKA/CREB pathway contributes to the analgesic effects of electroacupuncture in the anterior cingulate cortex in a rat pain memory model," Neural Plasticity, vol. 2016, Article ID 5320641, 16 pages, 2016. 
[29] Z. J. Weng, L. Y. Wu, C. L. Zhou et al., "Effect of electroacupuncture on $\mathrm{P} 2 \mathrm{X} 3$ receptor regulation in the peripheral and central nervous systems of rats with visceral pain caused by irritable bowel syndrome," Purinergic Signal, vol. 11, no. 3, pp. 321-329, 2015.

[30] Y. Qiao, F. Wu, J. Wang, X. Cui, C. Liu, and X. Zhu, "Effects of injection of anti-corticotropin release hormone serum in the lateral ventricles and electroacupuncture analgesia on pain threshold in rats with adjuvant arthritis," Neural Regeneration Research, vol. 7, no. 21, pp. 1630-1636, 2012.

[31] M. M. Heinricher, I. Tavares, J. L. Leith, and B. M. Lumb, "Descending control of nociception: specificity, recruitment and plasticity," Brain Research Reviews, vol. 60, no. 1, pp. 214-225, 2009.

[32] A. Kumar, H. Kaur, and A. Singh, "Neuropathic pain models caused by damage to central or peripheral nervous system," Pharmacological Reports, vol. 70, no. 2, pp. 206-216, 2018.

[33] J. Lisman, R. Yasuda, and S. Raghavachari, "Mechanisms of CaMKII action in long-term potentiation," Nature Reviews Neuroscience, vol. 13, no. 3, pp. 169-182, 2012.

[34] L. J. Zhou, Y. Zhong, W. J. Ren, Y. Y. Li, T. Zhang, and X. G. Liu, "BDNF induces late-phase LTP of C-fiber evoked field potentials in rat spinal dorsal horn," Experimental Neurology, vol. 212, no. 2, pp. 507-514, 2008.

[35] A. J. Todd, "Neuronal circuitry for pain processing in the dorsal horn," Nature Reviews Neuroscience, vol. 11, no. 12, pp. 823-836, 2010.

[36] L. J. Cole, K. L. Bennell, Y. Ahamed et al., "Determining brain mechanisms that underpin analgesia induced by the use of pain coping skills," Pain Medicine, vol. 19, no. 11, pp. 21772190, 2018.

[37] M. Zhuo, "Cortical excitation and chronic pain," Trends in Neurosciences, vol. 31, no. 4, pp. 199-207, 2008.

[38] S. Doolen, C. B. Blake, B. N. Smith, and B. K. Taylor, "Peripheral nerve injury increases glutamate-evoked calcium mobilization in adult spinal cord neurons," Molecular Pain, vol. 8, 2012.

[39] C. Ultenius, B. Linderoth, B. A. Meyerson, and J. Wallin, "Spinal NMDA receptor phosphorylation correlates with the presence of neuropathic signs following peripheral nerve injury in the rat," Neuroscience Letters, vol. 399, no. 1-2, pp. 85-90, 2006.

[40] C. K. Tong, E. J. Kaftan, and A. B. Macdermott, "Functional identification of NR2 subunits contributing to NMDA receptors on substance $\mathrm{P}$ receptor-expressing dorsal horn neurons," Molecular Pain, vol. 4, 2008.

[41] V. Neugebauer, "Metabotropic glutamate receptors-important modulators of nociception and pain behavior," Pain, vol. 98 , no. 1 , pp. 1-8, 2002.

[42] W. S. Zhao, Z. N. Jiang, H. Shi, L. L. Xu, Y. Yang, and Y. C. Wang, "Low-frequency electroacupuncture alleviates chronic constrictive injury-induced mechanical allodynia by inhibiting NR2B upregulation in ipsilateral spinal dorsal horn in rats," Chinese Journal of Integrative Medicine, vol. 25, no. 6, pp. 462-467, 2019.

[43] Y. S. Lee, J. H. Lee, I. S. Lee, and B. T. Choi, "Effects of electroacupuncture on spinal $\alpha$-amino-3-hydroxy-5-methyl-4-isoxazole propionic acid receptor in rats injected with complete Freund's adjuvant," Molecular Medicine Reports, vol. 8, no. 4, pp. 1130-1134, 2013.

[44] K. Han, A. Zhang, Y. Mo et al., "Islet-cell autoantigen 69 mediates the antihyperalgesic effects of electroacupuncture on inflammatory pain by regulating spinal glutamate receptor subunit 2 phosphorylation through protein interacting with C-kinase 1 in mice," Pain, vol. 160, no. 3, pp. 712-723, 2019.

[45] H. N. Kim, Y. R. Kim, J. Y. Jang, H. K. Shin, and B. T. Choi, "Electroacupuncture confers antinociceptive effects via inhibition of glutamate transporter downregulation in complete Freund's adjuvant-injected rats," Evidence-based Complementary and Alternative Medicine, vol. 2012, Article ID 643973, 11 pages, 2012.

[46] J. Zeng, L. Y. Cui, Y. Feng, and M. X. Ding, "Electroacupuncture relieves neuropathic pain via upregulation of glutamate transporters in the spinal cord of rats," Neuroscience Letters, vol. 620, pp. 38-42, 2016.

[47] J. Zhou, Y. Jin, R. Ma et al., "Electroacupuncture alleviates experimental chronic inflammatory pain by inhibiting calcium voltage-gated channel-mediated inflammation," Evidence-Based Complementary and Alternative Medicine, vol. 2020, Article ID 7061972, 10 pages, 2020.

[48] M. L. Hu, F. Y. Zhou, J. J. Liu, Y. Ding, J. M. Zhong, and M. X. Ding, "Electroacupuncture inhibits the activation of p38MAPK in the central descending facilitatory pathway in rats with inflammatory pain," Evidence-Based Complementary and Alternative Medicine, vol. 2017, Article ID 7531060, 10 pages, 2017.

[49] X. C. Yuan, B. Zhu, X. H. Jing et al., "Electroacupuncture potentiates cannabinoid receptor-mediated descending inhibitory control in a mouse model of knee osteoarthritis," Frontiers in Molecular Neuroscience, vol. 11, p. 112, 2018.

[50] J. Li, C. Fu, H. Liu et al., "Electroacupuncture attenuates hyperalgesia in rats withdrawn from chronic alcohol drinking via habenular mu opioid receptors," Alcoholism, Clinical and Experimental Research, vol. 41, no. 3, pp. 637-643, 2017.

[51] X. Meng, Y. Zhang, A. Li et al., "The effects of opioid receptor antagonists on electroacupuncture-produced anti-allodynia/hyperalgesia in rats with paclitaxel-evoked peripheral neuropathy," Brain Research, vol. 1414, pp. 58-65, 2011.

[52] C. P. Huang, Y. W. Lin, D. Y. Lee, and C. L. Hsieh, "Electroacupuncture relieves CCI-induced neuropathic pain involving excitatory and inhibitory neurotransmitters," EvidenceBased Complementary and Alternative Medicine, vol. 2019, Article ID 6784735, 9 pages, 2019.

[53] S. W. Jiang, Y. W. Lin, and C. L. Hsieh, "Electroacupuncture at Hua Tuo Jia Ji Acupoints reduced neuropathic pain and increased GABAA receptors in rat spinal cord," EvidenceBased Complementary and Alternative Medicine, vol. 2018, Article ID 8041820, 10 pages, 2018.

[54] S. S. Li, W. Z. Tu, C. Q. Jia et al., "KCC2-GABAA pathway correlates with the analgesic effect of electro-acupuncture in CCI rats," Molecular Medicine Reports, vol. 17, no. 5, pp. 6961-6968, 2018.

[55] J. W. Choi, S. Y. Kang, J. G. Choi et al., "Analgesic effect of electroacupuncture on paclitaxel-induced neuropathic pain via spinal opioidergic and adrenergic mechanisms in mice," The American Journal of Chinese Medicine, vol. 43, no. 1, pp. 57-70, 2015.

[56] J. Choi, C. Jeon, J. H. Lee et al., "Suppressive effects of bee venom acupuncture on paclitaxel-induced neuropathic pain in rats: mediation by spinal $\alpha 2$-Adrenergic receptor," Toxins, vol. 9, no. 11, p. 351, 2017.

[57] J. H. Kim, H. Y. Kim, K. Chung, and J. M. Chung, "Electroacupuncture reduces the evoked responses of the spinal dorsal 
horn neurons in ankle-sprained rats," Journal of Neurophysiology, vol. 105, no. 5, pp. 2050-2057, 2011.

[58] Y. Zhang, R. X. Zhang, M. Zhang et al., "Electroacupuncture inhibition of hyperalgesia in an inflammatory pain rat model: involvement of distinct spinal serotonin and norepinephrine receptor subtypes," British Journal of Anaesthesia, vol. 109, no. 2, pp. 245-252, 2012.

[59] J. R. da Silva, M. L. da Silva, and W. A. Prado, "Electroacupuncture at $2 / 100 \mathrm{hz}$ activates antinociceptive spinal mechanisms different from those activated by electroacupuncture at 2 and $100 \mathrm{hz}$ in responder rats," Evidence-Based Complementary and Alternative Medicine, vol. 2013, Article ID 205316, 14 pages, 2013.

[60] Y. Y. Wu, Y. L. Jiang, X. F. He et al., "5-HT in the dorsal raphe nucleus is involved in the effects of $100-\mathrm{Hz}$ electroacupuncture on the pain-depression dyad in rats," Experimental and Therapeutic Medicine, vol. 14, no. 1, pp. 107114, 2017.

[61] Y. Zhang, A. Li, J. Xin et al., "Involvement of spinal serotonin receptors in electroacupuncture anti-hyperalgesia in an inflammatory pain rat model," Neurochemical Research, vol. 36, no. 10, pp. 1785-1792, 2011.

[62] Y. Zhang, A. Li, J. Xin et al., "Electroacupuncture alleviates chemotherapy-induced pain through inhibiting phosphorylation of spinal CaMKII in rats," European Journal of Pain, vol. 22, no. 4, pp. 679-690, 2018.

[63] A. Li, Y. Zhang, L. Lao et al., "Serotonin receptor $2 \mathrm{~A} / \mathrm{C}$ is involved in electroacupuncture inhibition of pain in an osteoarthritis rat model," Evidence-Based Complementary and Alternative Medicine, vol. 2011, Article ID 619650, 6 pages, 2011.

[64] Y. Liang, Y. Qiu, J. du et al., "Inhibition of spinal microglia and astrocytes contributes to the anti-allodynic effect of electroacupuncture in neuropathic pain induced by spinal nerve ligation," Acupuncture in Medicine, vol. 34, no. 1, pp. 4047, 2016.

[65] D. C. Choi, J. Y. Lee, E. J. Lim, H. H. Baik, T. H. Oh, and T. Y. Yune, "Inhibition of ROS-induced p38MAPK and ERK activation in microglia by acupuncture relieves neuropathic pain after spinal cord injury in rats," Experimental Neurology, vol. 236, no. 2, pp. 268-282, 2012.

[66] J. Y. Lee, D. C. Choi, T. H. Oh, and T. Y. Yune, "Analgesic effect of acupuncture is mediated via inhibition of JNK activation in astrocytes after spinal cord injury," PLoS One, vol. 8, no. 9, article e73948, 2013.

[67] J. Wang, Y. Gao, S. Chen et al., "The effect of repeated electroacupuncture analgesia on neurotrophic and cytokine factors in neuropathic pain rats," Evidence-Based Complementary and Alternative Medicine, vol. 2016, Article ID 8403064, 11 pages, 2016.

[68] M. L. Yu, R. D. Wei, T. Zhang et al., "Electroacupuncture relieves pain and attenuates inflammation progression through inducing IL-10 production in CFA-induced mice," Inflammation, vol. 43, no. 4, pp. 1233-1245, 2020.

[69] W. J. Dai, J. L. Sun, C. Li et al., "Involvement of Interleukin10 in analgesia of electroacupuncture on incision pain," Evidence-Based Complementary and Alternative Medicine, vol. 2019, Article ID 8413576, 11 pages, 2019.

[70] U. Ali, E. Apryani, H. Y. Wu, X. F. Mao, H. Liu, and Y. X. Wang, "Low frequency electroacupuncture alleviates neuropathic pain by activation of spinal microglial IL- $10 / \beta$-endor- phin pathway," Biomedicine \& Pharmacotherapy, vol. 125, p. 109898, 2020.

[71] Y. X. Zhao, M. J. Yao, Q. Liu, J. J. Xin, J. H. Gao, and X. C. Yu, "Electroacupuncture treatment attenuates paclitaxel-induced neuropathic pain in rats via inhibiting spinal glia and the TLR4/NF- $\kappa$ B pathway," Journal of Pain Research, vol. Volume 13, pp. 239-250, 2020.

[72] H. C. Hsu, C. L. Hsieh, S. Y. Wu, and Y. W. Lin, "Toll-like receptor 2 plays an essential role in electroacupuncture analgesia in a mouse model of inflammatory pain," Acupuncture in Medicine, vol. 37, no. 6, pp. 356-364, 2019.

[73] Y. H. Gao, C. W. Li, J. Y. Wang et al., "Effect of electroacupuncture on the cervicospinal $\mathrm{P} 2 \mathrm{X} 7$ receptor/fractalkine/CX3CR1 signaling pathway in a rat neck-incision pain model," Purinergic Signal, vol. 13, no. 2, pp. 215-225, 2017.

[74] Y. Li, Z. Fang, N. Gu et al., "Inhibition of chemokine CX3CL1 in spinal cord mediates the electroacupuncture-induced suppression of inflammatory pain," Journal of Pain Research, vol. Volume 12, pp. 2663-2672, 2019.

[75] C. Li, W. Mao, Y. K. Huang, Z. Q. Zhao, and N. Lyu, "Roles of CX3CR1 in mediation of post-incision induced mechanical pain hypersensitivity: effects of acupuncture-combined anesthesia," Sheng Li Xue Bao, vol. 70, no. 3, pp. 237-244, 2018.

[76] Q. Hu, X. Zheng, X. Li et al., "Electroacupuncture alleviates mechanical allodynia in a rat model of complex regional pain syndrome type-I via suppressing spinal CXCL12/CXCR4 signaling," The Journal of Pain, vol. 21, no. 9-10, pp. 1060-1074, 2020.

[77] X. Yan and H. R. Weng, "Endogenous interleukin- $1 \beta$ in neuropathic rats enhances glutamate release from the primary afferents in the spinal dorsal horn through coupling with presynapticN-methyl-d-aspartic acid receptors," The Journal of Biological Chemistry, vol. 288, no. 42, pp. 30544-30557, 2013.

[78] M. Swartjes, A. Morariu, M. Niesters, L. Aarts, and A. Dahan, "Nonselective and NR2B-selective N-methyl-D-aspartic acid receptor antagonists produce antinociception and long-term relief of allodynia in acute and neuropathic pain," Anesthesiology, vol. 115, no. 1, pp. 165-174, 2011.

[79] D. L. Somers and F. R. Clemente, "Contralateral high or a combination of high- and low-frequency transcutaneous electrical nerve stimulation reduces mechanical allodynia and alters dorsal horn neurotransmitter content in neuropathic rats," The Journal of Pain, vol. 10, no. 2, pp. 221229, 2009.

[80] Y. Li, X. Zhang, H. Liu et al., "Retracted: phosphorylated CaMKII post-synaptic binding to NR2B subunits in the anterior cingulate cortex mediates visceral pain in visceral hypersensitive rats," Journal of Neurochemistry, vol. 121, no. 4, pp. 662-671, 2012.

[81] R. H. Dworkin, A. B. O'Connor, J. Audette et al., "Recommendations for the pharmacological management of neuropathic pain: an overview and literature update," Mayo Clinic Proceedings, vol. 85, no. 3, pp. S3-14, 2010.

[82] S. A. Kissiwaa, S. D. Patel, B. L. Winters, and E. E. Bagley, "Opioids differentially modulate two synapses important for pain processing in the amygdala," British Journal of Pharmacology, vol. 177, no. 2, pp. 420-431, 2020.

[83] M. Rodriguez-Munoz, P. Sanchez-Blazquez, A. Vicente-Sanchez, E. Berrocoso, and J. Garzon, "The mu-opioid receptor and the NMDA receptor associate in PAG neurons: implications in pain control," Neuropsychopharmacology, vol. 37, no. 2, pp. 338-349, 2012. 
[84] H. P. Li, W. Su, Y. Shu et al., "Electroacupuncture decreases Netrin-1-induced myelinated afferent fiber sprouting and neuropathic pain through $\mu$-opioid receptors," Journal of Pain Research, vol. Volume 12, pp. 1259-1268, 2019.

[85] H. Y. Liao, C. L. Hsieh, C. P. Huang, and Y. W. Lin, "Electroacupuncture attenuates induction of inflammatory pain by regulating opioid and adenosine pathways in mice," Scientific Reports, vol. 7, no. 1, p. 15679, 2017.

[86] S. Zhang, H. Tang, J. Zhou, and Y. Gu, "Electroacupuncture attenuates neuropathic pain after brachial plexus injury," Neural Regeneration Research, vol. 9, no. 14, pp. 1365-1370, 2014.

[87] Y. Zhang, A. Li, L. Lao et al., "Rostral ventromedial medulla $\mu$, but not $\kappa$, opioid receptors are involved in electroacupuncture anti-hyperalgesia in an inflammatory pain rat model," Brain Research, vol. 1395, pp. 38-45, 2011.

[88] T. Chen, W. W. Zhang, Y. X. Chu, and Y. Q. Wang, "Acupuncture for pain management: molecular mechanisms of action," The American Journal of Chinese Medicine, vol. 48, no. 4, pp. 793-811, 2020.

[89] H. Zhu, H. C. Xiang, H. P. Li, et al., "Inhibition of GABAergic neurons and excitation of glutamatergic neurons in the ventrolateral periaqueductal gray participate in electroacupuncture analgesia mediated by cannabinoid receptor," Frontiers in Neuroscience, vol. 13, p. 484, 2019.

[90] J. Yowtak, J. Wang, H. Y. Kim, Y. Lu, K. Chung, and J. M. Chung, "Effect of antioxidant treatment on spinal GABA neurons in a neuropathic pain model in the mouse," Pain, vol. 154, no. 11, pp. 2469-2476, 2013.

[91] A. François, S. A. Low, E. I. Sypek et al., “A brainstem-spinal cord inhibitory circuit for mechanical pain modulation by GABA and Enkephalins," Neuron, vol. 93, no. 4, pp. 822839.e6, 2017.

[92] D. Nutt, "GABAA receptors: subtypes, regional distribution, and function," Journal of Clinical Sleep Medicine, vol. 2, no. 2, pp. S7-11, 2006.

[93] L. Pu, N. Xu, P. Xia et al., "Inhibition of Activity of GABA Transporter GAT1 by -Opioid Receptor," Evidence-Based Complementary and Alternative Medicine, vol. 2012, Article ID 818451, 12 pages, 2012.

[94] R. Tamano, M. Ishida, T. Asaki, M. Hasegawa, and S. Shinohara, "Effect of spinal monoaminergic neuronal system dysfunction on pain threshold in rats, and the analgesic effect of serotonin and norepinephrine reuptake inhibitors," Neuroscience Letters, vol. 615, pp. 78-82, 2016.

[95] W. Rahman, C. S. Bauer, K. Bannister, J. L. Vonsy, A. C. Dolphin, and A. H. Dickenson, "Descending serotonergic facilitation and the antinociceptive effects of pregabalin in a rat model of osteoarthritic pain," Molecular Pain, vol. 5, pp. 1744-8069, 2009.

[96] M. Zhuo, "Descending facilitation," Molecular Pain, vol. 13, pp. 17448069-17699212, 2017.

[97] P. Pei, L. Liu, L. P. Zhao et al., "Electroacupuncture exerts an anti-migraine effect via modulation of the 5-HT7 receptor in the conscious rat," Acupuncture in Medicine, vol. 37, no. 1, pp. 47-54, 2019.

[98] J. C. Wu, E. T. Ziea, L. Lao et al., "Effect of electroacupuncture on visceral hyperalgesia, serotonin and fos expression in an animal model of irritable bowel syndrome," Journal of Neurogastroenterology and Motility, vol. 16, no. 3, pp. 306-314, 2010.
[99] L. Liu, P. Pei, L. P. Zhao, Z. Y. Qu, Y. P. Zhu, and L. P. Wang, "Electroacupuncture pretreatment at GB20 exerts antinociceptive effects via peripheral and central serotonin mechanism in conscious migraine rats," Evidence-Based Complementary and Alternative Medicine, vol. 2016, Article ID 1846296, 10 pages, 2016.

[100] V. Parpura, T. A. Basarsky, F. Liu, K. Jeftinija, S. Jeftinija, and P. G. Haydon, "Glutamate-mediated astrocyte-neuron signalling,” Nature, vol. 369, no. 6483, pp. 744-747, 1994.

[101] R. R. Ji, A. Nackley, Y. Huh, N. Terrando, and W. Maixner, "Neuroinflammation and central sensitization in chronic and widespread pain," Anesthesiology, vol. 129, no. 2, pp. 343-366, 2018.

[102] Y. M. Lau, S. C. Wong, S. W. Tsang, W. K. Lau, A. P. Lu, and H. Zhang, "Cellular sources of cyclooxygenase-1 and -2 upregulation in the spinal dorsal horn after spinal nerve ligation," Neuropathology and Applied Neurobiology, vol. 40, no. 4, pp. 452-463, 2014.

[103] M. L. Loggia, D. B. Chonde, O. Akeju et al., "Evidence for brain glial activation in chronic pain patients," Brain, vol. 138, no. 3, pp. 604-615, 2015.

[104] R. R. Ji, T. Berta, and M. Nedergaard, "Glia and pain: is chronic pain a gliopathy?," Pain, vol. 154, Supplement 1, pp. S10-S28, 2013.

[105] J. M. Pocock and H. Kettenmann, "Neurotransmitter receptors on microglia," Trends in Neurosciences, vol. 30, no. 10, pp. 527-535, 2007.

[106] D. Schomberg and J. K. Olson, "Immune responses of microglia in the spinal cord: contribution to pain states," Experimental Neurology, vol. 234, no. 2, pp. 262-270, 2012.

[107] J. Cao, J. S. Wang, X. H. Ren, and W. D. Zang, "Spinal sample showing P-JNK and P38 associated with the pain signaling transduction of glial cell in neuropathic pain," Spinal Cord, vol. 53, no. 2, pp. 92-97, 2015.

[108] Y. J. Gao, L. Zhang, O. A. Samad et al., "JNK-induced MCP-1 production in spinal cord astrocytes contributes to central sensitization and neuropathic pain," The Journal of Neuroscience, vol. 29, no. 13, pp. 4096-4108, 2009.

[109] Z. J. Zhang, D. L. Cao, X. Zhang, R. R. Ji, and Y. J. Gao, "Chemokine contribution to neuropathic pain: respective induction of CXCL1 and CXCR2 in spinal cord astrocytes and neurons," Pain, vol. 154, no. 10, pp. 2185-2197, 2013.

[110] M. Zhuo, G. Wu, and L. J. Wu, "Neuronal and microglial mechanisms of neuropathic pain," Molecular Brain, vol. 4, no. 1 , p. 31, 2011.

[111] H. Zhang, H. Zhang, and P. M. Dougherty, "Dynamic effects of TNF- $\alpha$ on synaptic transmission in mice over time following sciatic nerve chronic constriction injury," Journal of Neurophysiology, vol. 110, no. 7, pp. 1663-1671, 2013.

[112] B. Sung, G. Lim, and J. Mao, "Altered expression and uptake activity of spinal glutamate transporters after nerve injury contribute to the pathogenesis of neuropathic pain in rats," The Journal of Neuroscience, vol. 23, no. 7, pp. 2899-2910, 2003.

[113] C. J. Woolf and M. W. Salter, "Neuronal plasticity: increasing the gain in pain," Science, vol. 288 , no. 5472, pp. 1765-1768, 2000.

[114] G. Rajkowska and J. J. Miguel-Hidalgo, "Gliogenesis and glial pathology in depression," CNS \& Neurological Disorders Drug Targets, vol. 6, no. 3, pp. 219-233, 2007. 
[115] S. S. Ballon Romero, Y. C. Lee, L. J. Fuh, H. Y. Chung, S. Y. Hung, and Y. H. Chen, "Analgesic and neuroprotective effects of electroacupuncture in a dental pulp injury modela basic research," International Journal of Molecular Sciences, vol. 21, no. 7, p. 2628, 2020.

[116] W. Z. Tu, S. S. Li, X. Jiang et al., "Effect of electroacupuncture on the BDNF-TrkB pathway in the spinal cord of CCI rats," International Journal of Molecular Medicine, vol. 41, no. 6, pp. 3307-3315, 2018.

[117] J. Y. Wang, Y. H. Gao, L. N. Qiao et al., "Repeated electroacupuncture treatment attenuated hyperalgesia through suppression of spinal glial activation in chronic neuropathic pain rats," BMC Complementary and Alternative Medicine, vol. 18, no. 1, p. 74, 2018.

[118] J. Q. Fang, J. Y. Du, Y. Liang, and J. F. Fang, "Intervention of electroacupuncture on spinal p38 MAPK/ATF-2/VR-1 pathway in treating inflammatory pain induced by CFA in rats," Molecular Pain, vol. 9, p. 13, 2013.

[119] J. Q. Fang, J. F. Fang, Y. Liang, and J. Y. Du, "Electroacupuncture mediates extracellular signal-regulated kinase $1 / 2$ pathways in the spinal cord of rats with inflammatory pain," BMC Complementary and Alternative Medicine, vol. 14, no. 1, p. 285, 2014.

[120] P. Han, S. Liu, M. Zhang et al., "Inhibition of spinal interlukin-33/ST2 signaling and downstream ERK and JNK pathways in electroacupuncture analgesia in formalin mice," PLoS One, vol. 10, no. 6, article e0129576, 2015.

[121] Q. Liu, Y. Liu, J. Bian, Q. Li, and Y. Zhang, "The preemptive analgesia of pre-electroacupuncture in rats with formalininduced acute inflammatory pain," Molecular Pain, vol. 15, article 1744806919866529, 2019.

[122] W. Tu, W. Wang, H. Xi, R. He, L. Gao, and S. Jiang, "Regulation of Neurotrophin-3 and Interleukin-1 and Inhibition of Spinal Glial Activation Contribute to the Analgesic Effect of Electroacupuncture in Chronic Neuropathic Pain States of Rats," Evidence-based Complementary and Alternative Medicine, vol. 2015, Article ID 642081, 9 pages, 2015.

[123] J. W. Griffith, C. L. Sokol, and A. D. Luster, "Chemokines and chemokine receptors: positioning cells for host defense and immunity," Annual Review of Immunology, vol. 32, no. 1, pp. 659-702, 2014.

[124] P. E. Love and A. Bhandoola, "Signal integration and crosstalk during thymocyte migration and emigration," Nature Reviews. Immunology, vol. 11, no. 7, pp. 469-477, 2011.

[125] C. Savarin-Vuaillat and R. M. Ransohoff, "Chemokines and chemokine receptors in neurological disease: raise, retain, or reduce?," Neurotherapeutics, vol. 4, no. 4, pp. 590-601, 2007.

[126] J. M. Dawes, H. Kiesewetter, J. R. Perkins, D. L. Bennett, and S. B. McMahon, "Chemokine expression in peripheral tissues from the monosodium Lodoacetate model of chronic joint pain," Molecular Pain, vol. 9, pp. 1744-8069, 2013.

[127] M. de Miguel, D. C. Kraychete, and R. J. Meyer Nascimento, "Chronic pain: cytokines, lymphocytes and chemokines," Inflammation \& Allergy Drug Targets, vol. 13, no. 5, pp. 339-349, 2014.

[128] M. A. Thacker, A. K. Clark, F. Marchand, and S. B. McMahon, "Pathophysiology of peripheral neuropathic pain: immune cells and molecules," Anesthesia and Analgesia, vol. 105, no. 3, pp. 838-847, 2007.
[129] R. J. Miller, W. Rostene, E. Apartis et al., "Chemokine action in the nervous system," The Journal of Neuroscience, vol. 28, no. 46, pp. 11792-11795, 2008.

[130] A. Jaerve and H. W. Muller, "Chemokines in CNS injury and repair," Cell and Tissue Research, vol. 349, no. 1, pp. 229-248, 2012.

[131] J. H. Hu, J. P. Yang, L. Liu et al., "Involvement of CX3CR1 in bone cancer pain through the activation of microglia p38 MAPK pathway in the spinal cord," Brain Research, vol. 1465, pp. 1-9, 2012.

[132] Y. Q. Zhou, H. Y. Gao, X. H. Guan et al., "Chemokines and their receptors: potential therapeutic targets for bone cancer pain," Current Pharmaceutical Design, vol. 21, no. 34, pp. 5029-5033, 2015.

[133] Y. J. Gao and R. R. Ji, "Chemokines, neuronal-glial interactions, and central processing of neuropathic pain," Pharmacology \& Therapeutics, vol. 126, no. 1, pp. 56-68, 2010.

[134] Z. Y. Zhuang, Y. Kawasaki, P. H. Tan, Y. R. Wen, J. Huang, and R. R. Ji, "Role of the CX3CR1/p38 MAPK pathway in spinal microglia for the development of neuropathic pain following nerve injury-induced cleavage of fractalkine," Brain, Behavior, and Immunity, vol. 21, no. 5, pp. 642-651, 2007.

[135] K. M. Lee, S. M. Jeon, and H. J. Cho, "Interleukin-6 induces microglial CX3CR1 expression in the spinal cord after peripheral nerve injury through the activation of p38 MAPK," European Journal of Pain, vol. 14, no. 7, pp. 682.e1-682.e12, 2010.

[136] A. K. Clark, P. K. Yip, J. Grist et al., "Inhibition of spinal microglial cathepsin S for the reversal of neuropathic pain," Proceedings of the National Academy of Sciences of the United States of America, vol. 104, no. 25, pp. 10655-10660, 2007.

[137] E. Milligan, V. Zapata, D. Schoeniger et al., “An initial investigation of spinal mechanisms underlying pain enhancement induced by fractalkine, a neuronally released chemokine," The European Journal of Neuroscience, vol. 22, no. 11, pp. 2775-2782, 2005.

[138] A. K. Clark and M. Malcangio, "Fractalkine/CX3CR1 signaling during neuropathic pain," Frontiers in Cellular Neuroscience, vol. 8, 2014.

[139] N. D. Jayaraj, B. J. Bhattacharyya, A. A. Belmadani et al., "Reducing CXCR4-mediated nociceptor hyperexcitability reverses painful diabetic neuropathy," The Journal of Clinical Investigation, vol. 128, no. 6, pp. 2205-2225, 2018.

[140] X. Luo, W. L. Tai, L. Sun et al., "Crosstalk between astrocytic CXCL12 and microglial CXCR4 contributes to the development of neuropathic pain," Molecular Pain, vol. 12, p. $174480691663638,2016$.

[141] R. D. Gosselin, C. Varela, G. Banisadr et al., "Constitutive expression of CCR 2 chemokine receptor and inhibition by MCP-1/CCL2 of GABA-induced currents in spinal cord neurones," Journal of Neurochemistry, vol. 95, no. 4, pp. $1023-$ 1034, 2005.

[142] D. Spicarova, P. Adamek, N. Kalynovska, P. Mrozkova, and J. Palecek, "TRPV1 receptor inhibition decreases CCL2induced hyperalgesia," Neuropharmacology, vol. 81, pp. 7584, 2014.

[143] D. L. Cao, Z. J. Zhang, R. G. Xie, B. C. Jiang, R. R. Ji, and Y. J. Gao, "Chemokine CXCL1 enhances inflammatory pain and increases NMDA receptor activity and COX-2 expression in spinal cord neurons via activation of CXCR2," Experimental Neurology, vol. 261, pp. 328-336, 2014. 
[144] J. Xu, M. D. Zhu, X. Zhang et al., "NF $\kappa B$-mediated CXCL1 production in spinal cord astrocytes contributes to the maintenance of bone cancer pain in mice," Journal of Neuroinflammation, vol. 11, no. 1, p. 38, 2014.

[145] L. Cao and J. T. Malon, "Anti-nociceptive role of CXCL1 in a murine model of peripheral nerve injury-induced neuropathic pain," Neuroscience, vol. 372, pp. 225-236, 2018.

[146] W. Guo, S. Imai, J. L. Yang et al., "In vivo immune interactions of multipotent stromal cells underlie their long-lasting pain-relieving effect," Scientific Reports, vol. 7, no. 1, p. 10107, 2017. 